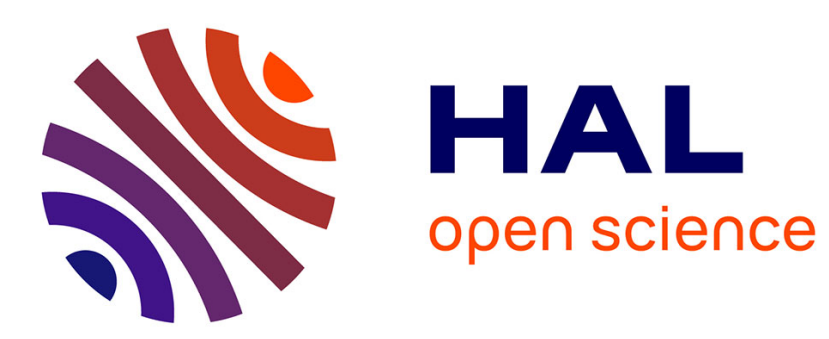

\title{
A Mathematical Model of the Paris Metro Pricing Scheme for Charging Packet Networks
}

\author{
David Ros, Bruno Tuffin
}

\section{To cite this version:}

David Ros, Bruno Tuffin. A Mathematical Model of the Paris Metro Pricing Scheme for Charging

Packet Networks. [Research Report] RR-4775, INRIA. 2003. inria-00071811

\section{HAL Id: inria-00071811 \\ https://hal.inria.fr/inria-00071811}

Submitted on 23 May 2006

HAL is a multi-disciplinary open access archive for the deposit and dissemination of scientific research documents, whether they are published or not. The documents may come from teaching and research institutions in France or abroad, or from public or private research centers.
L'archive ouverte pluridisciplinaire HAL, est destinée au dépôt et à la diffusion de documents scientifiques de niveau recherche, publiés ou non, émanant des établissements d'enseignement et de recherche français ou étrangers, des laboratoires publics ou privés. 
INSTITUT NATIONAL DE RECHERCHE EN INFORMATIQUE ET EN AUTOMATIQUE

\section{A Mathematical Model of the Paris Metro Pricing Scheme for Charging Packet Networks}

David Ros, Bruno Tuffin

\section{$\mathbf{N}^{\circ} \mathbf{4 7 7 5}$}

Mars 2003

THÈME 1 



\title{
RINRIA
}

\section{A Mathematical Model of the Paris Metro Pricing Scheme for Charging Packet Networks}

\author{
David Ros*, Bruno Tuffin ${ }^{\dagger}$ \\ Thème 1 - Réseaux et systèmes \\ Projet Armor \\ Rapport de recherche $\mathrm{n}^{\circ} 4775$ - Mars 2003 - 42 pages
}

\begin{abstract}
Pricing has become one of the main challenges of the networking community and is receiving a lot of interest in the literature. In this paper, we analyze the so-called Paris Metro Pricing scheme which separates the network into different and independent subnetworks, each behaving equivalently, except that they charge their customers at different rates. In our model, each subnetwork is represented by a single bottleneck queue, and the "customers" (data packets) choose their subnetwork taking into account not only the prices, but also the expected delay, which is supposed to have an economic impact. We obtain some necessary and sufficient conditions for the stability of the system; we analyze the problem of maximizing the network revenue and compare it with the case of a single network, and present several extensions of the model. Numerical results illustrating some key aspects of the system are provided throughout the paper.
\end{abstract}

Key-words: Internet, packet networks, pricing, queueing models.

(Résumé : tsvp)

* David.Ros@enst-bretagne.fr

$\dagger$ Bruno.Tuffin@irisa.fr

Unité de recherche INRIA Rennes

IRISA, Campus universitaire de Beaulieu, 35042 RENNES Cedex (France)

Téléphone : 0299847100 - International : +33299847100

Télécopie : 0299847171 - International : +33299847171 


\section{Un modèle mathématique du schéma de tarification du métro de Paris appliqué aux réseaux}

Résumé : La tarification est devenue l'un des pricipaux défis de la communauté des réseaux et reçoit actuellement beaucoup d'attention dans la littérature. Dans cet article, nous analysons la méthode dite de tarification du métro parisien qui sépare le réseau en différents sous-réseaux indépendents agissant à l'identique, mais à un coût différent. Dans notre modèle, les sous-réseaux sont représentés par un unique goulot d'étranglement et les clients (des paquets de données) choisissent leur réseau selon le prix, mais aussi le délai moyen qui est supposé avoir un impact économique. Nous obtenons des conditions nécessaires et suffisantes pour la stabilité du système ; nous analysons le problème de la maximisation du revenu du réseau et comparons avec le cas d'un unique réseau, enfin nous donnons plusieurs extensions du modèle. De nombreux exemples sont fournis tout au long du papier afin d'illustrer nos propos.

Mots-clé : Internet, réseaux de paquets, tarification, files d'attente. 


\section{Introduction}

Pricing in IP networks is becoming a main concern of Internet Service Providers (ISPs). Classical pricing schemes, based on a subscription fee and/or a (possibly unlimited) number of hours of network access, depending on the country and the type of usage, are becoming obsolete due to three major reasons:

1. Such simple schemes were an incentive to stimulate utilization. Even if they probably helped to develop the network and allowed ISPs to gain market share, nowadays they may prove sub-optimal. Indeed, demand in access networks may easily exceed the installed capacity, leading to congestion.

2. Some users might wish to pay more than others to avoid congestion and get an improved service. Why not serve them first and (possibly) increase the ISP revenue?

3. Unlike the telephone network, the current Internet - and, more so, the next-generation Internet - has to deal with different kinds of applications with diverse quality of service (QoS) requirements:

- Interactive video needs very small delays and fairly low packet losses. On the other hand, high-quality video-on-demand may tolerate a higher delay and jitter (within some bounds) but lower packet losses than interactive video.

- Voice over IP requires small delays but can afford some packet losses.

- Electronic mail can afford delay (within some bounds).

- File transfer needs a good average throughput, whereas web browsing demands also a relatively low latency.

- Remote login requires small round-trip times.

Including these requirements in a pricing scheme may, in some way, improve users' satisfaction.

A wide range of charging methods for packet networks has been devised and discussed in the literature; see for instance the survey papers $[2,3,5,8,12$,

$\mathrm{RR} \quad \mathrm{n}^{\circ} 4775$ 
14]. The main classes include resource reservation, priorities between packets, auctions for bandwidth or pricing based on transfer rates.

In this paper, we study another method, called Paris Metro Pricing (PMP) and introduced by A. Odlyzko in $[6,10]$. Under the PMP scheme, the network is split into independent subnetworks; more precisely, a fixed fraction of the capacity of each link is totally allocated to each subnetwork. The tariff will be different for each subnetwork, so that (hopefully) congestion will be alleviated in the most expensive ones. This method does not offer any QoS guarantees, so that it is somehow weak with respect to the abovementioned techniques; however, it is very attractive because of its implementation and management simplicity. In this paper, we develop a mathematical model which may be helpful in setting prices in a PMP-based network. Particularly, our model will use the delay introduced by the network as a cost. Even if it has been argued that network segmentation might not work under competition [7] (i.e., no Nash equilibrium exists), this result applies to a model quite different from ours. Note also that in [4], PMP has been adapted in such a way that, instead of logically separating the subnetworks, a round-robin service discipline is used to share the bandwidth among them. This "improved" version of PMP, even though it deserves attention, is not considered here.

The layout of the paper is as follows. The PMP scheme is introduced in Section 2. In Section 3 the mathematical model is described. Necessary and sufficient conditions for stability are then provided, and an optimization problem (maximizing the ISP revenue) is introduced. Section 4 provides some numerical illustrations of the results of Section 3. In Section 5, we compare the revenues of a PMP network with those of an equivalent, non-PMP network. Section 6 is devoted to some extensions of the model: the case of multiple applications with different delay sensitivities, the case where the performance measures of interest include the loss probabilities, and time-of-day pricing. Finally, conclusions are given in Section 7.

\section{PMP: a brief description}

The original PMP proposal in [10] consists in partitioning a network into several logically separate networks (or classes), each having a fixed fraction of 
the capacity of the entire network. Every subnetwork would route and handle packets according to the current Internet protocols. There is no formal guarantee of QoS, but by charging different rates for different classes (served in the same way), it is supposed that the most expensive classes will be less congested by way of self-regulation, hence delivering a better QoS. The name given to this model, Paris Metro Pricing, stems from the rules of the Paris Metro about 20 years ago, where trains were composed of cars of two classes, offering exactly the same quality of seats. As tickets prices were different, the cars for the most expensive class were less congested, leading to a better perceived QoS.

As pointed out by Odlyzko, the advantage of PMP is that, even if we do not have any strict QoS guarantee using this scheme, but rather a statistical guarantee, it would permit dispensing with complex, non-scalable mechanisms (like, say, resource reservation protocols) and keep the simpler and cheaper current model of the Internet, while improving the QoS experience. Indeed, no signalling protocols would have to be used, and the tariff would be fixed, which is preferred by most users [11].

From the preceding discussion, it is intuitively clear that prices play in PMP an important role in controlling congestion and, notably, in distributing the load among subnetworks: prices are the key to quality-of-service differentiation. The problem of finding the "right" set of prices for a given bandwidth partition may be informally stated as follows: for a PMP network to work in an efficient manner ${ }^{1}$, the charge for using the most expensive subnetwork should be high enough, so that this subnetwork is lightly loaded, but not "too high" - otherwise, the subnetwork would remain empty, because the high quality offered would not compensate for the (very) high monetary cost incurred by users.

In the next section, we present a mathematical model of PMP that allows us to analyze its stability properties with respect to prices, as well as to solve the revenue maximization problem.

\footnotetext{
${ }^{1}$ Efficiency may be defined, for instance, in terms of optimizing the network operator's revenue and providing an adequate usage of each subnetwork's resources.
}

$\mathrm{RR} \mathrm{n}^{\circ} 4775$ 


\section{Mathematical model}

\subsection{Model presentation}

Assume that there are $I$ classes (i.e., subnetworks) in a PMP network, and that the class- $i$ per-packet price at the entrance of the network is $p_{i}(1 \leq i \leq I)$.

We assume that there is a potential total arrival rate $\tilde{\lambda}$ of packets at the network, corresponding to the arrival rate when prices are set to 0 .

A utility measure is associated to each packet. It is assumed to be a random variable $U$ that follows the same distribution for every packet, and that it is independent of other packets' utility.

Also, a total cost function

$$
p_{i}+\gamma d_{i}
$$

is associated to a class $i$, where $d_{i}$ is the mean delay for a packet in the network and $\gamma$ is a constant converting delay in money. A packet enters (i.e., chooses) network $i$ if $i=\operatorname{argmin}_{j} p_{j}+\gamma d_{j}$ and $U \geq p_{i}+\gamma d_{i}$, that is, it chooses the less expensive subnetwork in terms of total cost. If $U<\min _{j} p_{j}+\gamma d_{j}$, the packet does not enter at all, meaning that the network is too expensive for him. Traffic elasticity is then a central assumption of our model since a larger delay can be accepted by a user, provided that the price is decreased proportionally.

The actual total arrival rate is then

$$
\lambda=\tilde{\lambda} P\left(U \geq \min _{j} p_{j}+\gamma d_{j}\right)
$$

We define by $\bar{F}$ the remaining cumulative distribution function of $U$, i.e., $\bar{F}(x)=P(U \geq x)$. Finally, denote by $\lambda_{i}(1 \leq i \leq I)$ the actual arrival rate at subnetwork $i$, so that $\lambda=\sum_{i=1}^{I} \lambda_{i}$.

\subsection{Stability: existence and uniqueness}

Using this model, in equilibrium, the distribution of packets among classes has to be stable, meaning that the total cost $p_{j}+\gamma d_{j}$ is the same for all classes $j$. Indeed, if for a given class $j$ the value $p_{j}+\gamma d_{j}$ were smaller than the total cost of the other classes, then new packets entering the network would choose class $j$ until its total cost reaches that of other classes. This corresponds to 
a Wardrop equilibrium [1]. Let us call $p_{\text {tot }}$ the value $p_{j}+\gamma d_{j}$ (identical for all $j$ ). As we want all subnetworks to be used, we have the following set of $I+1$ equations with $I+1$ unknown variables $\lambda_{i}(1 \leq i \leq I)$ and $p_{t o t}$ :

$$
\left\{\begin{array}{l}
\sum_{i=1}^{I} \lambda_{i}=\tilde{\lambda} P\left(U \geq p_{\text {tot }}\right) \\
p_{i}+\gamma d_{i}=p_{\text {tot }}, \text { for } 1 \leq i \leq I
\end{array}\right.
$$

where $d_{i}$ is a function of $\lambda_{i}$.

The remaining of this section is separated into two parts. In the first part, the $d_{i}$ represent the mean waiting times (i.e., service excluded). In the second part, results are obtained as a corollary when the $d_{i}$ represent the response times (i.e., service included). What users and applications care about is total delay, so the latter case seems more realistic; nonetheless, we will begin by treating the waiting time case for the sake of clarity.

In order to determine $p_{\text {tot }}$ and $\lambda_{i} \forall i$, we have the following theorem.

Theorem 1. Assume that $d_{i}=f_{i}\left(\lambda_{i}\right) \in \mathbb{R}^{+}$is a strictly increasing and continuous function of the arrival rate, representing the mean waiting time of a class-i user. Without loss of generality, we suppose that $p_{1}>p_{2}>\cdots>p_{I}$. Assume also that the distribution of $U$ is absolutely continuous and strictly increasing. Then the solution of Eq. (1) exists and is unique if and only if

$$
p_{1} \leq \bar{F}^{-1}\left(\frac{1}{\tilde{\lambda}} \sum_{i=1}^{I} f_{i}^{-1}\left(\frac{p_{1}-p_{i}}{\gamma}\right)\right) .
$$

Remark 1. Note that if condition (2) is not verified, then it means that the highest price $p_{1}$ is too high to obtain an equilibrium between classes. Consequently, this class would be ignored (i.e., the subnetwork would remain empty) and the computation would have to be carried out again with the I-1 remaining classes.

Thus, condition (2) has to be taken as an assumption to get the equilibrium for I classes.

Proof of the theorem: Denote by $\bar{F}$ the remaining cumulative distribution function of random variable $U$. The system (1) can be re-written as

$$
\left\{\begin{aligned}
p_{t o t} & =\bar{F}^{-1}\left(\frac{1}{\tilde{\lambda}} \sum_{i=1}^{I} \lambda_{i}\right) \\
\lambda_{i} & =f_{i}^{-1}\left(\frac{p_{t o t}-p_{i}}{\gamma}\right), \text { for } 1 \leq i \leq I .
\end{aligned}\right.
$$

$\mathrm{RR} \mathrm{n}^{\circ} 4775$ 
In particular we can get

$$
p_{t o t}=\bar{F}^{-1}\left(\frac{1}{\tilde{\lambda}} \sum_{i=1}^{I} f_{i}^{-1}\left(\frac{p_{t o t}-p_{i}}{\gamma}\right)\right) .
$$

If we are able to prove that there exists a unique $p_{\text {tot }}$ satisfying this equation, then the existence and unicity of the $\lambda_{i}$ will be straightforward to show.

It is important to note that, by definition, $p_{t o t} \geq p_{i} \forall i$ (i.e., $p_{t o t} \geq p_{1}$ ) because $d_{i} \geq 0$.

Since $f_{i}^{-1}$ is strictly increasing $\forall i$ and $\bar{F}^{-1}$ is strictly decreasing (and both are continuous), $\bar{F}^{-1}\left(\frac{1}{\grave{\lambda}} \sum_{i=1}^{I} f_{i}^{-1}\left(\frac{p_{t o t}-p_{i}}{\gamma}\right)\right)$ is a continuous and strictly decreasing function of $p_{t o t}$. Thus the solution of (3), if it exists, is unique.

Existence depends on border values. Let $E\left(S_{i}\right)$ denote the mean service time for class $i$. The delay goes to infinity when the mean arrival rate approaches the mean service rate $1 / E\left(S_{i}\right)$, hence: $\lim _{d_{i} \rightarrow \infty} f_{i}^{-1}\left(d_{i}\right)=1 / E\left(S_{i}\right)$. Therefore, since the left-hand side of (3) tends to infinity and the right-hand side tends to $\bar{F}^{-1}\left(\frac{1}{\lambda} \sum_{i=1}^{I} \frac{1}{E\left(S_{i}\right)}\right)$ when $p_{t o t}$ tends to infinity, the existence depends on whether, at the minimal value of $p_{\text {tot }}$ (i.e., $p_{1}$ ), the right hand side of (3) is greater than or equal to $p_{1}$. Both situations are represented in Fig. 1. The condition is expressed by Eq. (2). In this case, Eq. (3) has a unique solution.

Corollary 1. Assume now that $d_{i}=f_{i}^{(R)}\left(\lambda_{i}\right)$ represents the system's response time. Then $d_{i} \in\left[E\left(S_{i}\right), \infty\right)$, with $E\left(S_{i}\right)$ denoting the mean service time.

In this case, and without loss of generality, the classes are supposed to be ordered by their minimum total cost $p_{1}+\gamma E\left(S_{1}\right) \geq p_{2}+\gamma E\left(S_{2}\right) \geq \cdots \geq$ $p_{I}+\gamma E\left(S_{I}\right)$.

Then the solution of (1) exists and is unique if and only if

$$
p_{1}+\gamma E\left(S_{1}\right) \leq \bar{F}^{-1}\left(\frac{1}{\tilde{\lambda}} \sum_{i=1}^{I}\left(f_{i}^{(R)}\right)^{-1}\left(\frac{p_{1}-p_{i}}{\gamma}+E\left(S_{1}\right)\right)\right),
$$

or equivalently, using the waiting times,

$$
p_{1}+\gamma E\left(S_{1}\right) \leq \bar{F}^{-1}\left(\frac{1}{\tilde{\lambda}} \sum_{i=1}^{I} f_{i}^{-1}\left(\frac{p_{1}-p_{i}}{\gamma}+E\left(S_{1}\right)-E\left(S_{i}\right)\right)\right),
$$




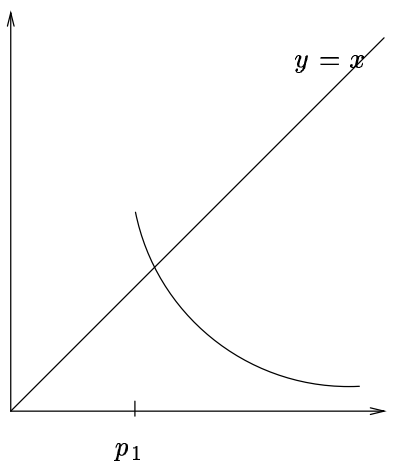

(i)

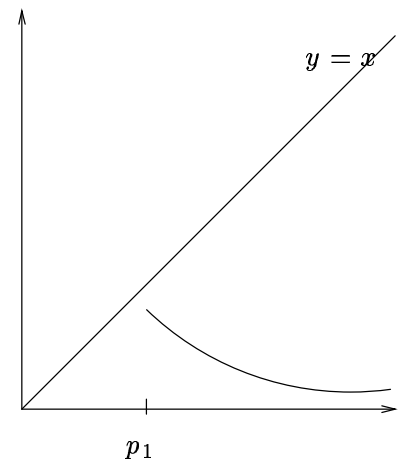

(ii)

Figure 1: Two situations for the border value. The solution (intersection of $y=\bar{F}^{-1}\left(\frac{1}{\tilde{\lambda}} \sum_{i=1}^{I} f_{i}^{-1}\left(\frac{x-p_{i}}{\gamma}\right)\right)$ with the $y=x$ line) exists in case (i) only.

Proof: Since the mean waiting time cannot be negative, we have to use the fact that $p_{\text {tot }} \geq \max _{i} p_{i}+\gamma E\left(S_{i}\right)=p_{1}+\gamma E\left(S_{1}\right)$. By replacing $p_{1}$ in Eq. (2) using $f_{i}^{(R)}$ instead of $f_{i}$, we get Eq. (4).

Besides, using the fact that $f_{i}^{(R)}$ is given by $f_{i}^{(R)}=f_{i}+E\left(S_{i}\right)$, we can easily obtain the equation with the waiting times.

Remark 2 (Special case: upper-bounded utility). In the case where the users' utility is bounded by a maximum utility $U_{\max }$ (that is, $\bar{F}(x)=0 \forall x \geq$ $\left.U_{\text {max }}\right)$, we can derive a simpler, necessary condition for stability as follows.

The inequality can be satisfied (meaning that some customers will enter the network) if and only if $\bar{F}\left(p_{1}+\gamma E\left(S_{1}\right)\right)>0$, but this can only be true if $p_{1}+\gamma E\left(S_{1}\right)<U_{\max }$. Hence, in order for the system to be stable, prices must satisfy the following condition:

$$
U_{\max }>p_{i}+\gamma E\left(S_{i}\right), \forall i
$$

Notice that $U_{\max } \leq \gamma E\left(S_{i}\right)$ means the cost due to fixed delay only is so high that class $i$ would never be chosen by users, regardless of the price $p_{i}$.

$\mathrm{RR} \mathrm{n}^{\circ} 4775$ 
Remark 3. It is easy to show that, for any I-tuple of prices $\left(p_{1}, \ldots, p_{I}\right)$ such that $p_{i}+\gamma E\left(S_{i}\right)=p_{j}+\gamma E\left(S_{j}\right) \forall i \neq j$, a unique solution of (1) exists as long as the utility is not upper-bounded by a finite $U_{\text {max }}$. We will call $\mathcal{E}_{0}$ the set of such prices.

Remark 4. Finally, note that (4) can be written as:

$$
p_{1}+\gamma E\left(S_{1}\right) \leq \bar{F}^{-1}\left(\frac{1}{\tilde{\lambda}} \sum_{i=2}^{I}\left(f_{i}^{(R)}\right)^{-1}\left(\frac{p_{1}-p_{i}}{\gamma}+E\left(S_{1}\right)\right)\right)
$$

because $\left(f_{1}^{(R)}\right)^{-1}\left(E\left(S_{1}\right)\right)=0$.

\subsection{Optimization problem}

The idea, from a network provider's perspective, is to find the $p_{i}$ maximizing the revenue of the network, i.e.,

$$
R=\max _{p_{i}, \forall i} \sum_{i=1}^{I} \lambda_{i} p_{i}
$$

subject to $p_{i} \geq 0 \forall i$.

Based on the existence and unicity condition of the arrival rates and total cost (defined in terms of $p_{i}$ ), and using the previous notations, the problem can be reformulated as:

$$
\max _{p_{i}, \forall i} \sum_{i=1}^{I} f_{i}^{-1}\left(\frac{p_{t o t}-p_{i}}{\gamma}\right) p_{i}
$$

subject to

$$
\begin{aligned}
p_{t o t} & \geq p_{i} \forall i \\
p_{t o t} & =\bar{F}^{-1}\left(\frac{1}{\tilde{\lambda}} \sum_{i=1}^{I} f_{i}^{-1}\left(\frac{p_{t o t}-p_{i}}{\gamma}\right)\right) \\
p_{i} & \geq 0 \forall i
\end{aligned}
$$


or its equivalent form, if we rather talk about the response time instead of the waiting time.

As this problem looks analytically intractable in the general case, in the next section we are going to present numerical results illustrating the stability domain, as well as the impact of prices and bandwidth partition on delay, total cost and revenue.

Remark that, in some sense, our approach can be related to that of Honig and Stieglitz [9] (albeit applied to a different model), where revenue, prices and performance were studied.

\section{Numerical results}

In this section we will present some numerical results obtained with the above model $^{2}$. In what follows we will use the response time of the system as a measure of delay, that is, $d_{i}=f^{(R)}\left(\lambda_{i}\right)$. We will also relax the restriction $p_{1}+\gamma E\left(S_{1}\right) \geq p_{2}+\gamma E\left(S_{2}\right)$ imposed in the previous section.

For the sake of simplicity, let us consider the case in which the ISP partitions its network in $I=2$ subnetworks. Indeed, even if the case $I>2$ is, in principle, not harder to solve numerically than the $I=2$ case, we will only consider the latter in order to being able of graphically presenting the results.

Each subnetwork $i$ is viewed as a single bottleneck, modeled as a M/G/1 queue with service rate $\mu_{i}=1 / E\left(S_{i}\right)$, so:

$$
f_{i}^{(R)}\left(\lambda_{i}\right)=\frac{1}{\mu_{i}}+\frac{\left(1+\mathrm{CV}^{2}\right) \rho_{i}}{2 \mu_{i}\left(1-\rho_{i}\right)}
$$

where $\rho_{i}=\lambda_{i} / \mu_{i}$ and $\mathrm{CV}^{2}$ is the squared coefficient of variation of the service law.

Unless stated otherwise, the following parameters will be used throughout this section:

- Total capacity of the network: $c=2$.

\footnotetext{
${ }^{2}$ The standard numerical and optimization packages included in, say, Matlab or Mathematica can be used to solve this problem.
}

$\mathrm{RR} \quad \mathrm{n}^{\circ} 4775$ 
- Potential total arrival rate: $\tilde{\lambda}=3$.

- Mean utility: $\bar{U}=1$.

- Cost per unit of delay: $\gamma=1$.

- Service time distribution: exponential (i.e., each queue is a $M / M / 1$ queue and so $\left.\mathrm{CV}^{2}=1\right)$. Note that the $\mathrm{M} / \mathrm{D} / 1$ queue gives similar results.

For comparison purposes, we have chosen two different distribution functions $\bar{F}$ for the utility: an exponential distribution $\exp (-u / \bar{U})$ and a uniform distribution in $\left[0, U_{\max }\right]$, where $U_{\max }=2 \bar{U}$. We assume, without loss of generality, that the total bandwidth $c$ of the network is shared among subnetworks according to:

$$
\mu_{1}=\alpha c, \mu_{2}=(1-\alpha) c \text { with } 0.5 \leq \alpha<1 .
$$

\subsection{Equilibrium region}

It is interesting to look at the shape of the equilibrium region, defined as the set $\mathcal{E}$ of all pairs $\left(p_{1}, p_{2}\right)$ such that the system of equations (1) has a unique solution, that is:

$$
\mathcal{E}=\mathcal{E}_{0} \cup \mathcal{E}_{1} \cup \mathcal{E}_{2}
$$

with $\mathcal{E}_{0}$ defined by the set of prices such that $p_{1}+\gamma / \mu_{1}=p_{2}+\gamma / \mu_{2}$ that also verify the stability condition and, for $i \in\{1,2\}$ :

$$
\mathcal{E}_{i}=\left\{\left(p_{1}, p_{2}\right): p_{-i}+\frac{\gamma}{\mu_{-i}}<p_{i}+\frac{\gamma}{\mu_{i}} \leq \bar{F}^{-1}\left(\frac{1}{\tilde{\lambda}}\left(f_{-i}^{(R)}\right)^{-1}\left(\frac{p_{i}-p_{-i}}{\gamma}+\frac{1}{\mu_{i}}\right)\right)\right\}
$$

where $-i$ denotes the element of $\{1,2\}$ which is not $i$. $\mathcal{E}_{i}$, for $i \in\{1,2\}$, can be regarded as the price area such that the charge plus the cost of service, i.e., the total cost minus the waiting cost, is more expensive for class $i$ (and such that the stability condition is verified). 


\subsubsection{Exponentially-distributed utility}

Figure 2 shows the equilibrium region for four distinct bandwidth allocations, in the case where the utility is exponentially distributed. The equilibrium region is shown in gray; the straight line pointed to by an arrow corresponds to the "frontier" $\mathcal{E}_{0}$ where the total costs (minus the waiting cost) are the same for both classes.

Note how the equilibrium region above the frontier gets narrower as the parameter $\alpha$ increases. This may be intuitively interpreted as follows: for a given $p_{1}$, as the service rate $\mu_{2}$ of subnetwork 2 gets lower (and so $\mu_{1}$ gets higher), subnetwork 1 would tend to attract more traffic than subnetwork 2 ; hence, total delay $d_{1}$ would eventually tend to increase so, for a fixed $p_{1}$, the maximum value $p_{2}$ can take must get lower in order to attain an equilibrium.

Remark also that, as prices get higher and higher, the equilibrium region degenerates into the straight line given by the frontier condition, no matter the value of $\alpha$. Since there is no upper bound to the utility (meaning that some users are willing to tolerate a very high cost), and the fixed cost $p_{i}+\gamma / \mu_{i}$ increases for both subnetworks, queueing delay tends to zero (together with the arrival rate), so equilibrium can only be reached if the fixed cost is about the same in each subnetwork; otherwise, traffic would switch entirely to the less-expensive one.

\subsubsection{Uniformly-distributed utility}

Figure 3 shows the equilibrium region for the same bandwidth allocations as in Fig. 2, but with a uniformly-distributed utility (with $U_{\max }=2$ ).

First, note that values of $p_{i}$ cannot be arbitrarily high because of the equilibrium condition (5). For instance, for $\alpha=0.6$ we have that $p_{1}<U_{\max }-\gamma / \mu_{1} \approx$ 1.167 and $p_{2}<U_{\max }-\gamma / \mu_{2}=0.75$ in order to have stability.

Moreover, remark that if $\alpha \geq 0.75$ equilibrium cannot be attained, irrespective of the prices. This is so because, in this case, $U_{\max } \leq \gamma / \mu_{2}$; in other words, the fixed delay of subnetwork 2 is higher than the highest delay users can tolerate.

$\mathrm{RR} \mathrm{n}^{\circ} 4775$ 

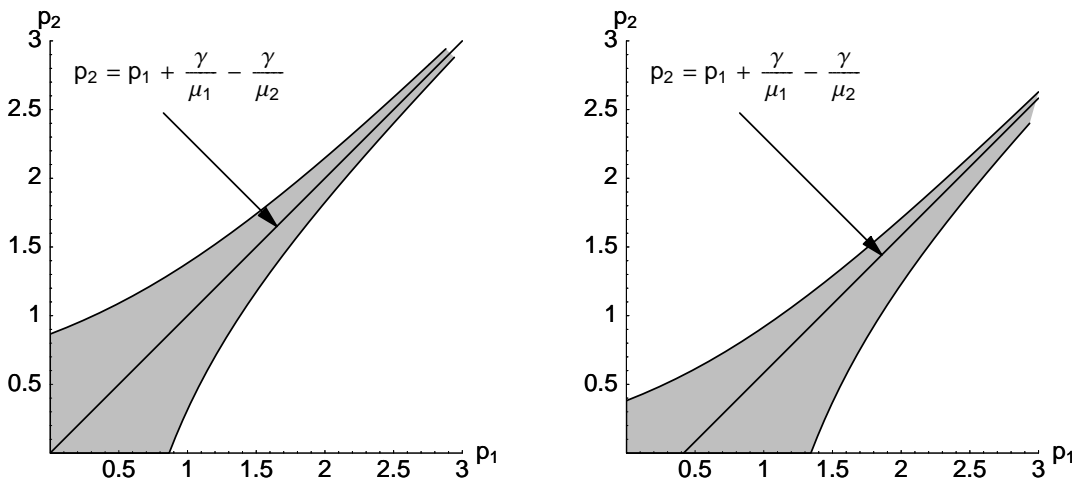

(a) $\alpha=0.5$

(b) $\alpha=0.6$
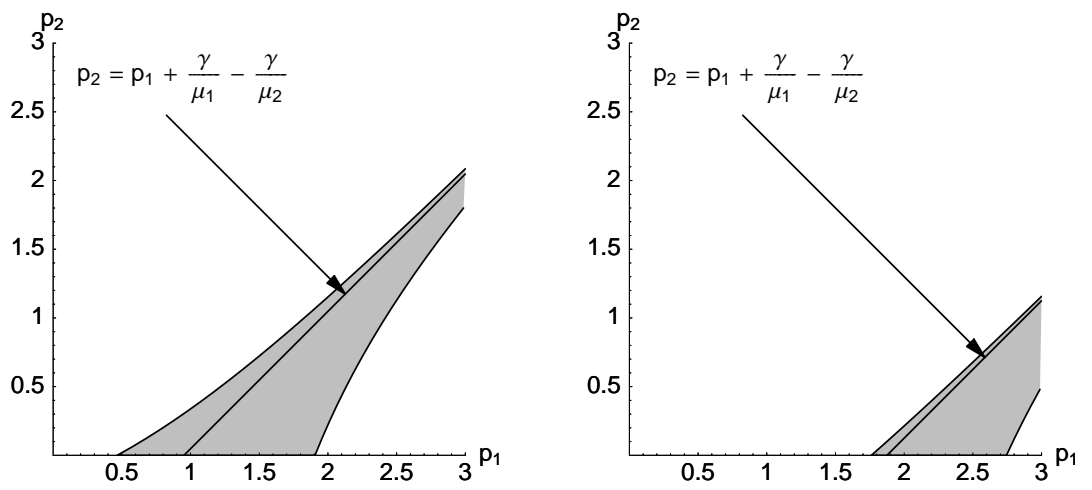

(c) $\alpha=0.7$

(d) $\alpha=0.8$

Figure 2: Equilibrium region, $\bar{F}$ exponential. 


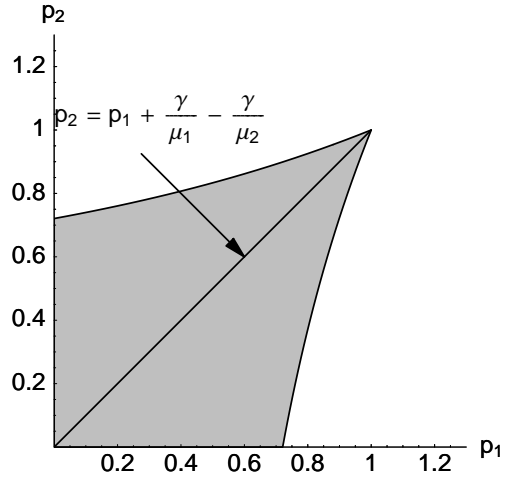

(a) $\alpha=0.5$

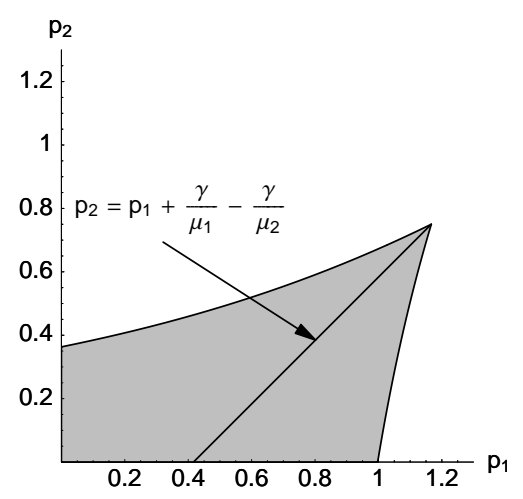

(b) $\alpha=0.6$

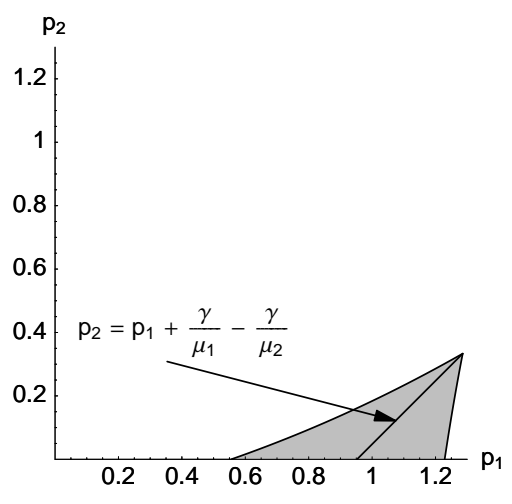

(c) $\alpha=0.7$

Figure 3: Equilibrium region, $\bar{F}$ uniform. 


\subsection{Total delay}

\subsubsection{Exponentially-distributed utility}

Delay in each subnetwork is shown in Figures 4 and 5. Observe that, for subnetwork $i$, delay is always minimal along the frontier of the equilibrium region $\mathcal{E}_{i}$.

The tradeoff between prices and delay is fairly evident in these figures. When one of the prices (say, $p_{1}$ ) is kept fixed, delay in the corresponding subnetwork (i.e., $\left.d_{1}\right)$ decreases as the other subnetwork's price $\left(p_{2}\right.$, in this case) decreases, while delay $d_{2}$ increases. This is due to the fact that a lower $p_{2}$ will tend to attract more customers to subnetwork 2, increasing queueing delay in it - and so decreasing queueing delay in subnetwork 1.

\subsubsection{Uniformly-distributed utility}

Figures 6 and 7 show the results obtained in the case of a uniformly-distributed utility. The same remarks as above can be done.

\subsection{Equilibrium cost}

\subsubsection{Exponentially-distributed utility}

Figure 8 shows that the total price $p_{\text {tot }}$ is strictly increasing with $p_{i}$. This, indeed, can easily be proved analytically.

\subsubsection{Uniformly-distributed utility}

The same remarks can be done in the uniform case, see Fig. 9.

\subsection{Revenue and optimality}

\subsubsection{Exponentially-distributed utility}

Figure 10 shows the revenue of the network, $R$, as a function of prices, for three different bandwidth allocations. It is interesting to remark that there appears to be a single maximum (i.e., there is a single pair of prices which is optimal, in the sense that revenue is maximized). As $\alpha$ increases, the position 


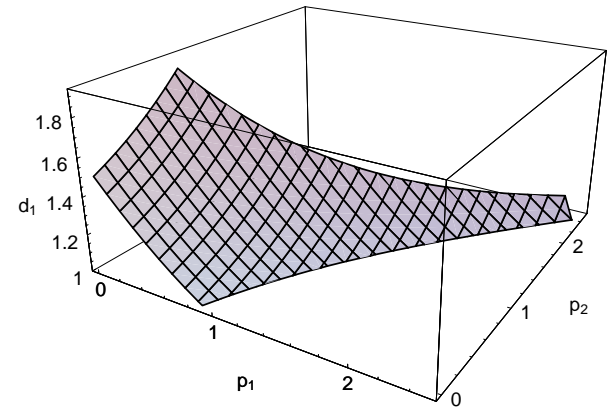

(a) $\alpha=0.5$

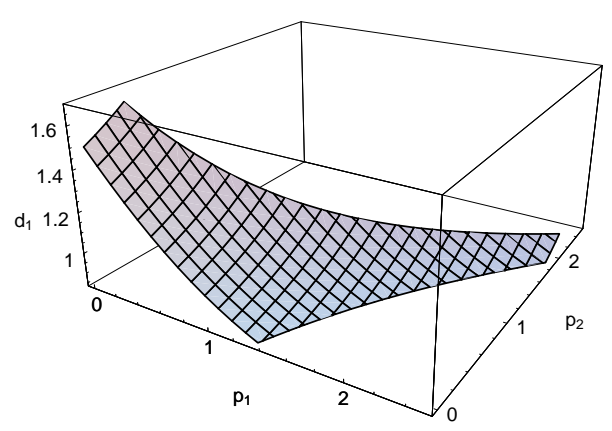

(b) $\alpha=0.6$

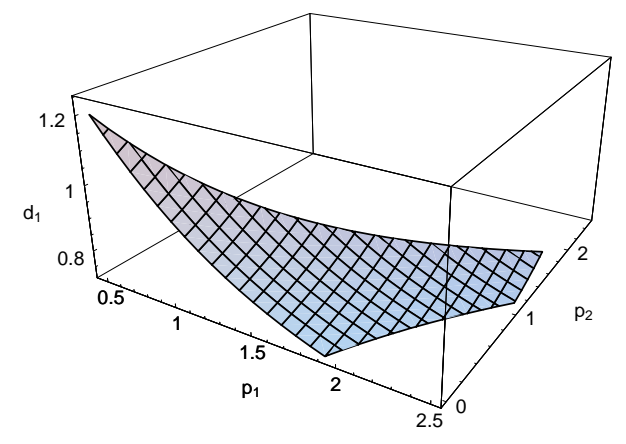

(c) $\alpha=0.7$

Figure 4: Delay in subnetwork $1, \bar{F}$ exponential.

$\mathrm{RR} \mathrm{n}^{\circ} 4775$ 


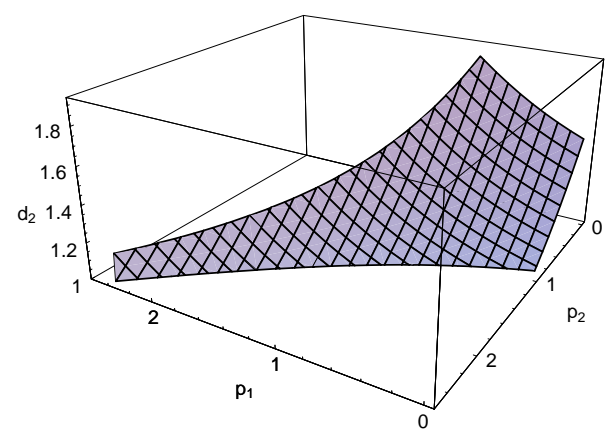

(a) $\alpha=0.5$

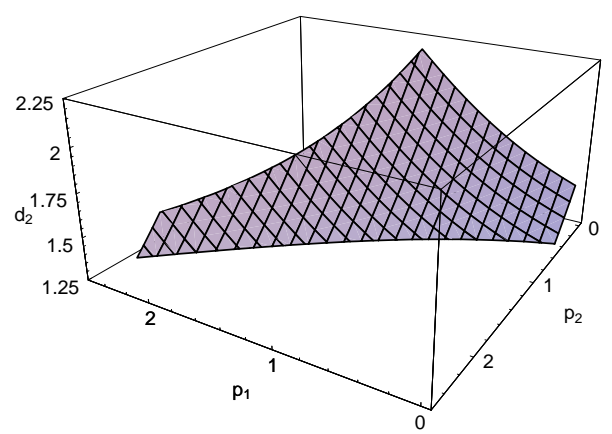

(b) $\alpha=0.6$

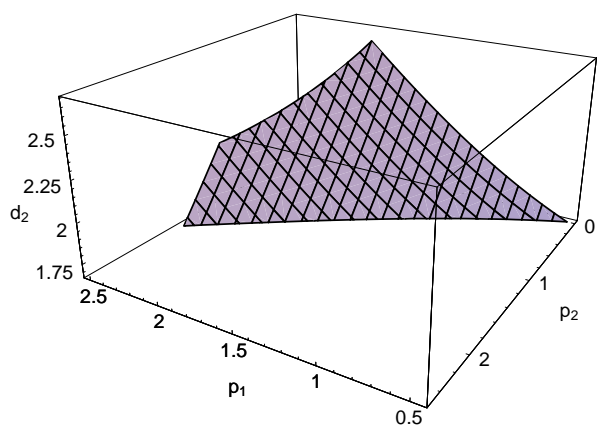

(c) $\alpha=0.7$

Figure 5: Delay in subnetwork $2, \bar{F}$ exponential. 


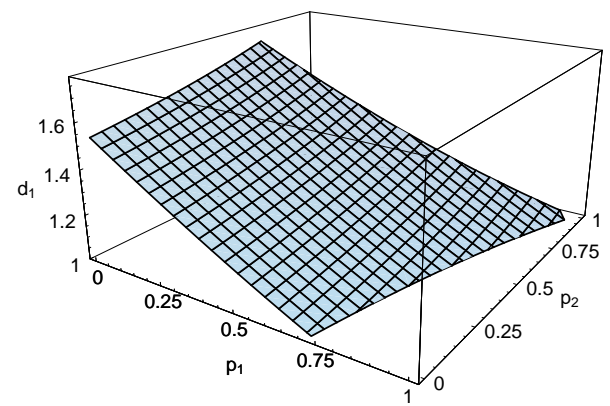

(a) $\alpha=0.5$

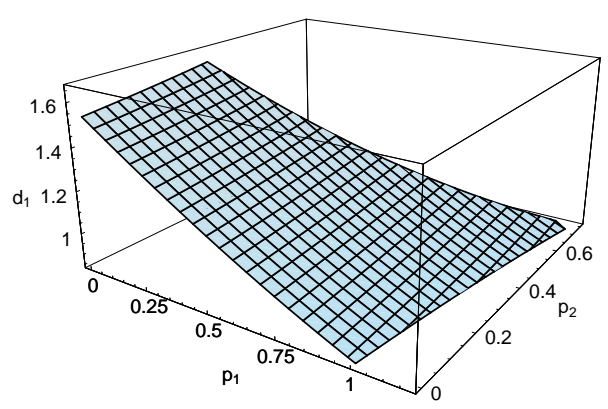

(b) $\alpha=0.6$

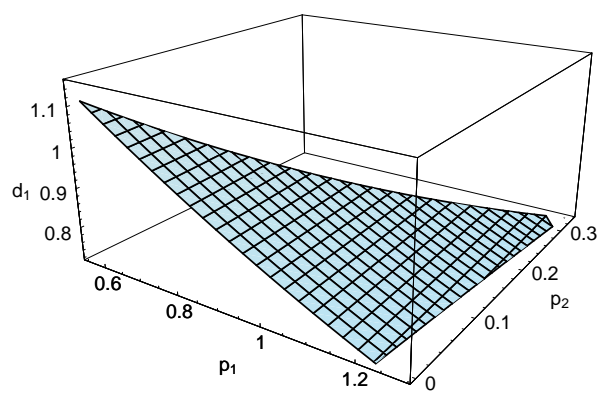

(c) $\alpha=0.7$

Figure 6: Delay in subnetwork $1, \bar{F}$ uniform.

$\mathrm{RR} \mathrm{n}^{\circ} 4775$ 


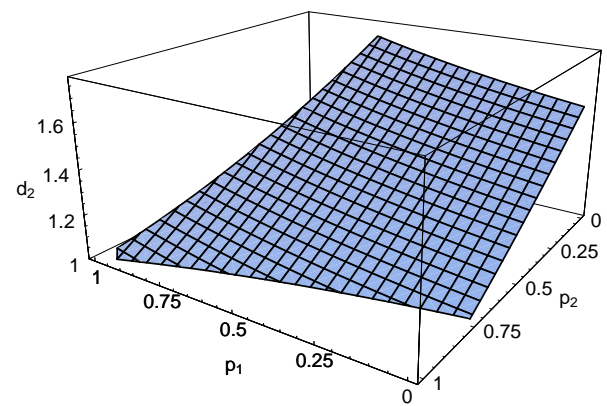

(a) $\alpha=0.5$

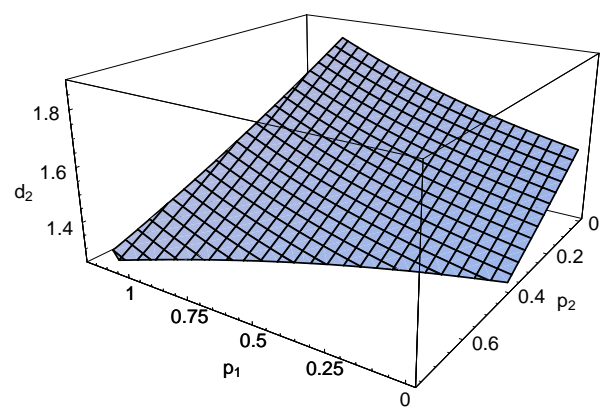

(b) $\alpha=0.6$

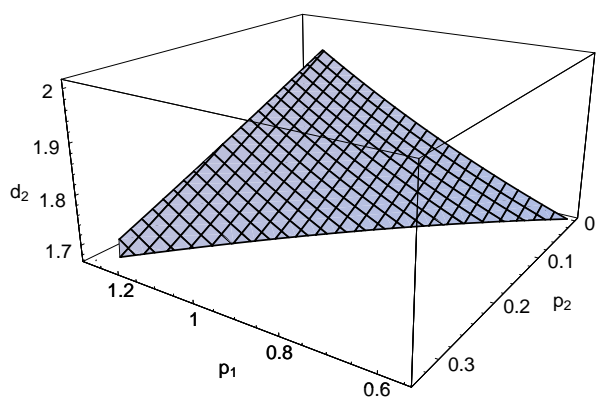

(c) $\alpha=0.7$

Figure 7: Delay in subnetwork 2, $\bar{F}$ uniform. 


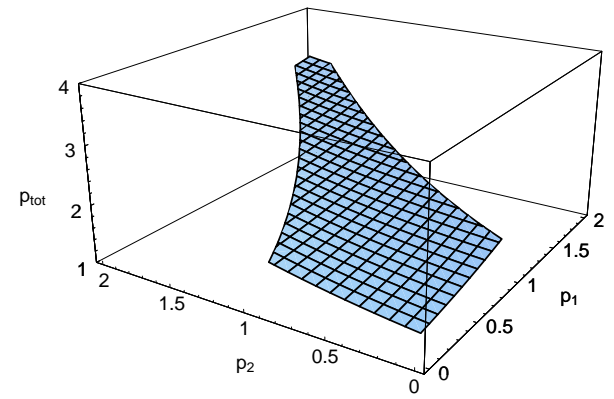

(a) $\alpha=0.5$

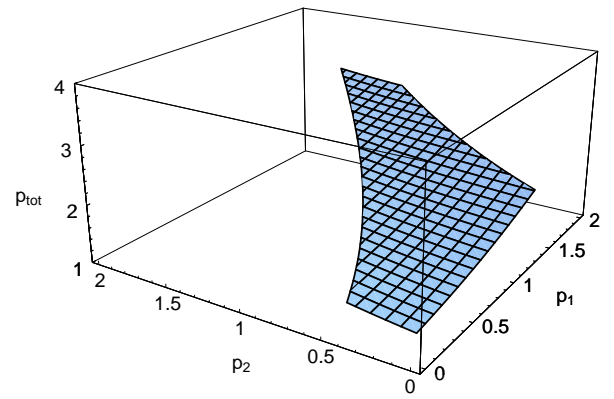

(b) $\alpha=0.6$

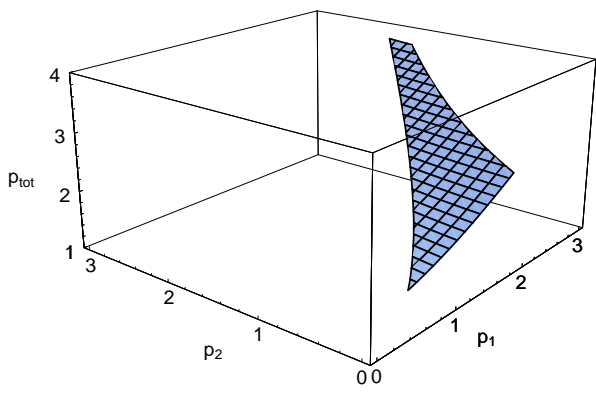

(c) $\alpha=0.7$

Figure 8: Equilibrium cost, $\bar{F}$ exponential.

$\mathrm{RR} \mathrm{n}^{\circ} 4775$ 


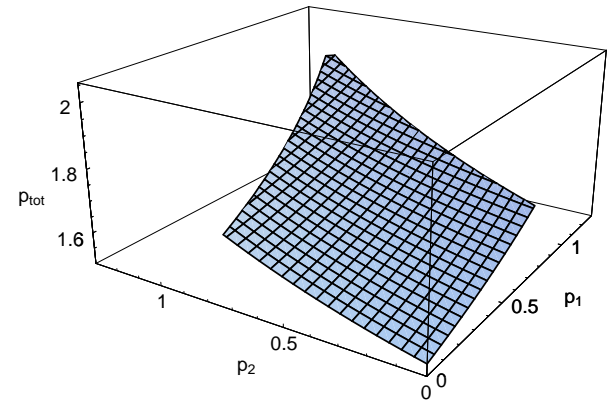

(a) $\alpha=0.5$

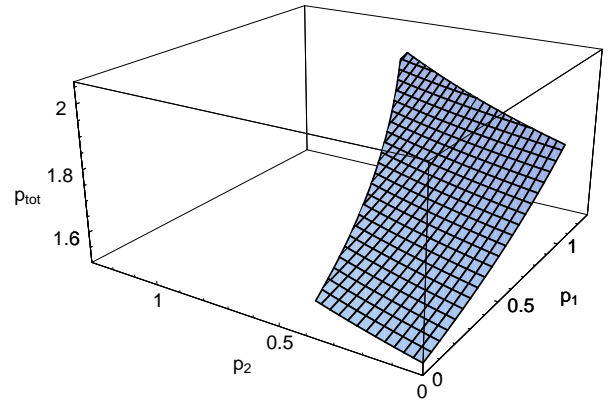

(b) $\alpha=0.6$

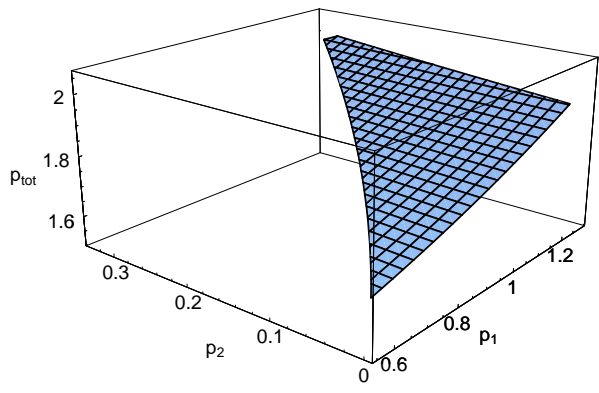

(c) $\alpha=0.7$

Figure 9: Equilibrium cost, $\bar{F}$ uniform. 
of the maximum value of $R$ moves closer to the rear "edge" of the surface, which corresponds to the upper edge of the equilibrium region $\mathcal{E}_{2}$ in Figure 2.

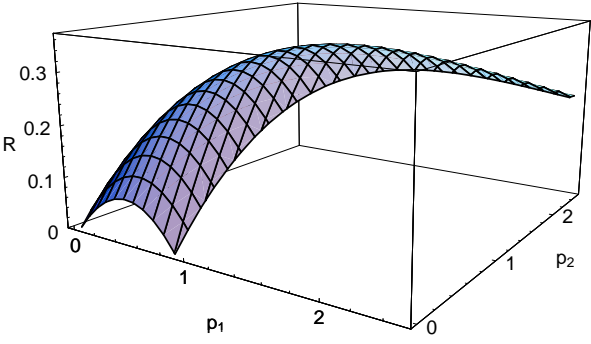

(a) $\alpha=0.5$

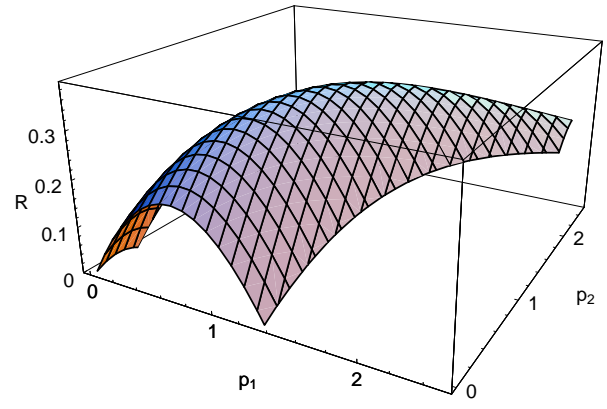

(b) $\alpha=0.6$

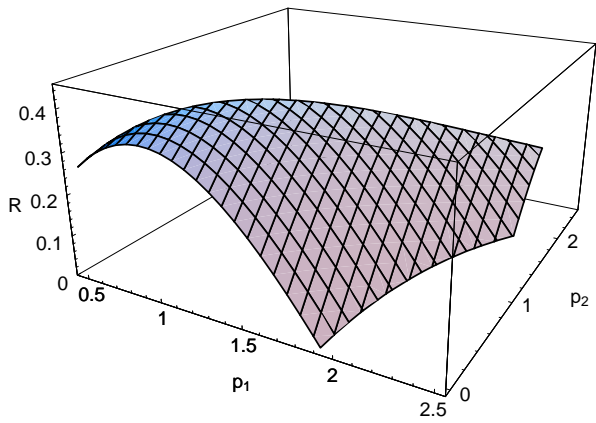

(c) $\alpha=0.7$

Figure 10: Revenue, $\bar{F}$ exponential.

\subsubsection{Uniformly-distributed utility}

The same remarks can be done in the case of a uniformly-distributed utility (see Fig. 11), where the same kind of behaviour is observed.

$\mathrm{RR} \mathrm{n}^{\circ} 4775$ 


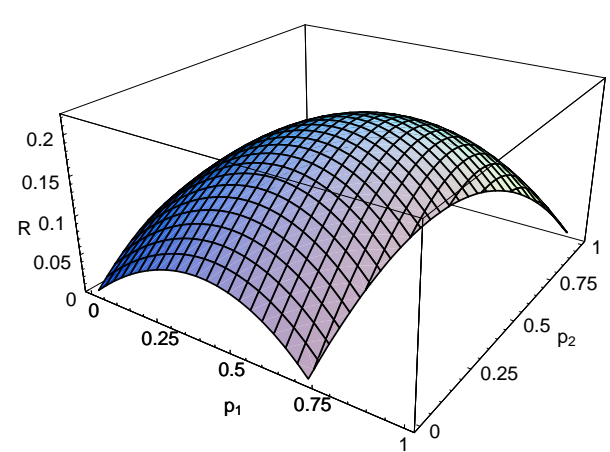

(a) $\alpha=0.5$

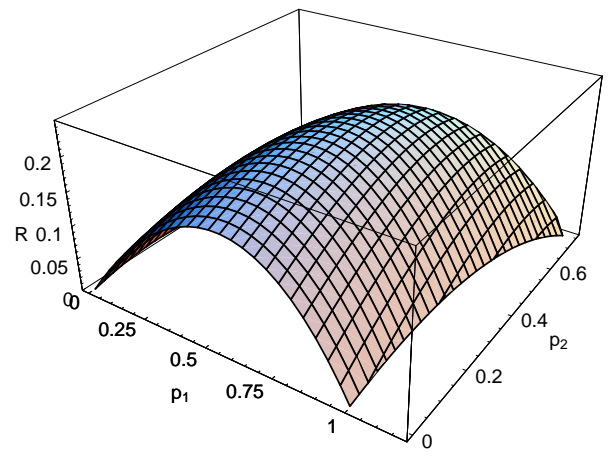

(b) $\alpha=0.6$

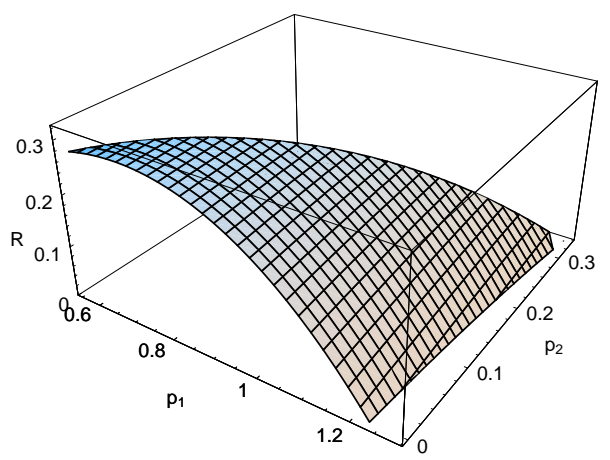

(c) $\alpha=0.7$

Figure 11: Revenue, $\bar{F}$ uniform. 


\subsection{Sensitivity of the optimal revenue}

\subsubsection{Sensitivity to the bandwidth partition}

Let us now examine how the optimal revenue, as well as the corresponding prices, delay and total cost, depend on the bandwidth allocation parameter $\alpha$. Figures 12 and 13 illustrate this for two distinct utility distribution functions with the same mean $\bar{U}=1$; all remaining parameters $(\gamma, \tilde{\lambda}$, and so on $)$ were kept unchanged.

First, remark that the optimal revenue $R_{\text {opt }}=\max _{p_{1}, p 2 \geq 0} R$ is highly sensitive to the value of the bandwidth allocation parameter $\alpha$, regardless of the type of distribution used for $U$. Figures 12(a) and 13(a) suggest that the ISP may find interesting, from an economic point of view, to operate at the maximum-revenue allocation $\alpha_{\text {opt }}$. However, since $R_{\text {opt }}$ (and, hence, the revenue for any non-optimal set of prices) rapidly decreases for values of $\alpha$ above $\alpha_{\text {opt }}$, a too-unequal bandwidth allocation policy may result in lower income. On the other hand, a "safe" bandwidth allocation (say, $\alpha=0.5$ ) may result in a maximum revenue as low as $\approx 66 \%$ of the highest $R_{o p t}$, in the case of Figure 13(a).

Note that, interestingly enough, the optimal revenue is maximized when the price $p_{2}$ of the lowest-capacity subnetwork drops to zero. For higher values of $\alpha$, since $p_{2}$ is at its lowest-possible value, the ISP cannot compensate for the poorer performance of subnetwork 2 by lowering its price, so more and more traffic tends to flow through subnetwork 1 and the price $p_{1}$ must be raised in order to have a stable network. For $\alpha<\alpha_{\text {opt }}$, a falling $p_{2}$ tends to attract more traffic to subnetwork 2 in spite of the degradation in delay $d_{2}$ when $\alpha$ increases, resulting in an increasing revenue.

\subsubsection{Sensitivity to the users' valuation of delay}

The $\gamma$ parameter, which expresses the cost per unit of delay, can be regarded as how much users value having a good quality of service (in terms of delay): the higher the value of $\gamma$, the higher the impact of delay on the total cost $p_{i}+\gamma d_{i}$, and the lower the probability that a given packet enters the network.

$\mathrm{RR} \mathrm{n}^{\circ} 4775$ 


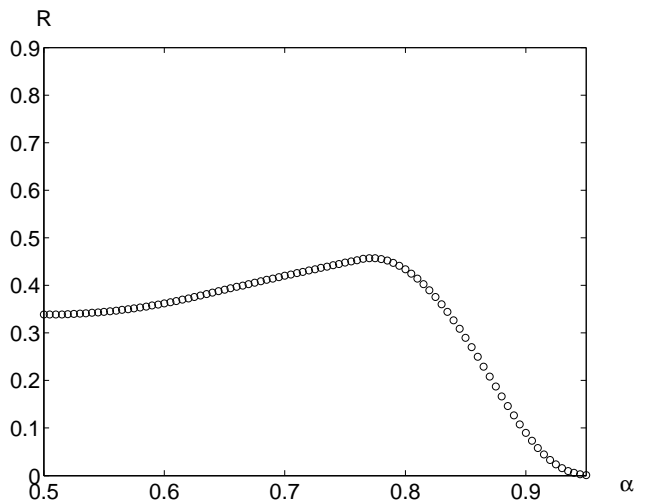

(a) Optimal revenue $R_{\text {opt }}$

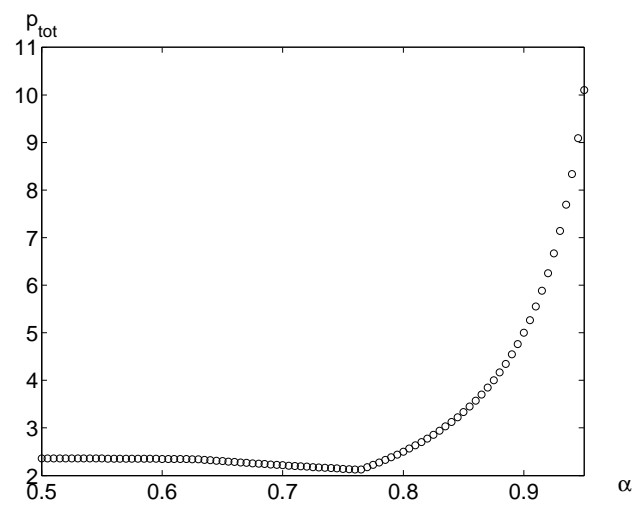

(c) Total cost $p_{t o t}$

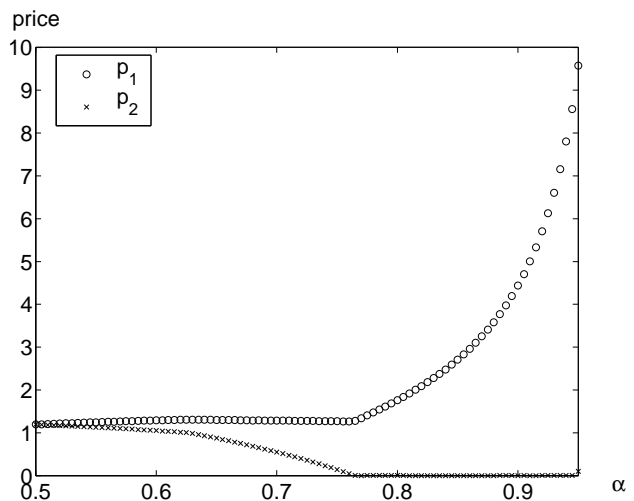

(b) Optimal prices $p_{i}$

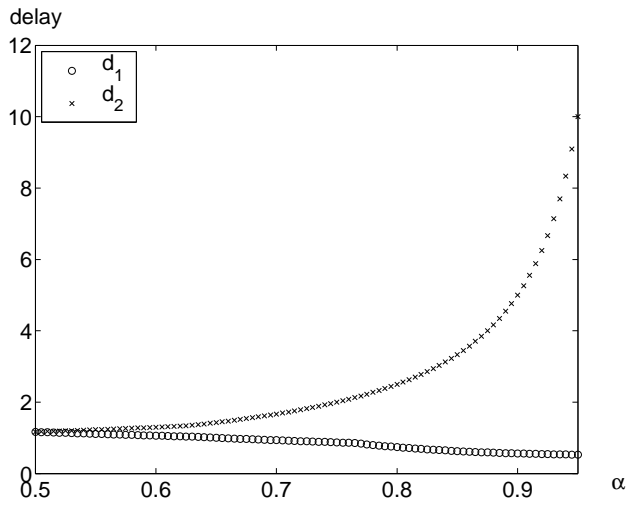

(d) Delay $d_{i}$

Figure 12: Optimal revenue and prices (and corresponding total cost and delay) as a function of $\alpha$, with an exponentially-distributed utility. 


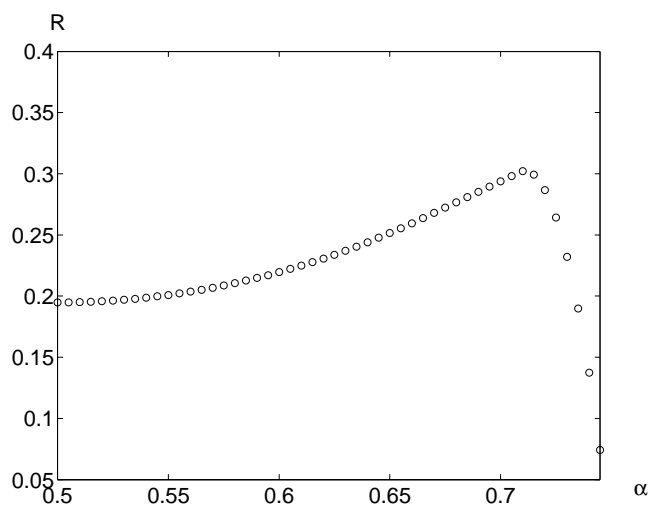

(a) Optimal revenue $R_{\text {opt }}$

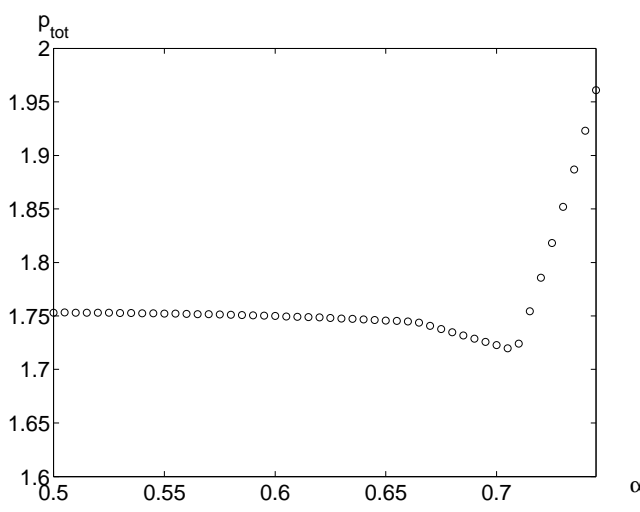

(c) Total cost $p_{t o t}$

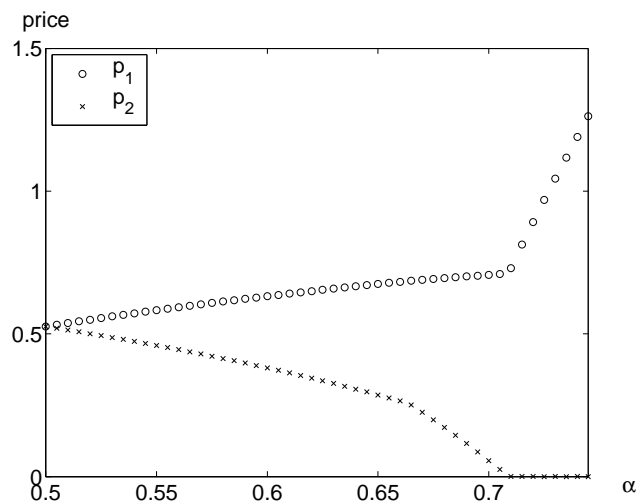

(b) Optimal prices $p_{i}$

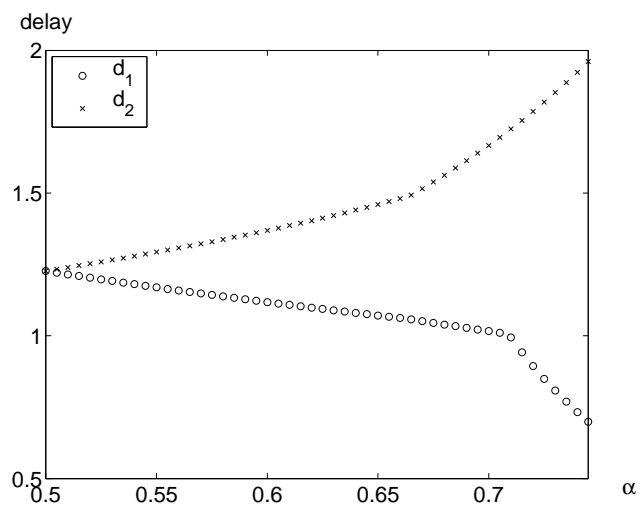

(d) Delay $d_{i}$

Figure 13: Optimal revenue and prices (and corresponding total cost and delay) as a function of $\alpha$, with an uniformly-distributed utility.

$\mathrm{RR} \mathrm{n}^{\circ} 4775$ 
Figure 14 illustrates the sensitivity of the optimal revenue to the value of $\gamma$, for $\alpha$ in the [0.5, 0.95] range. For $\gamma=0.25, R_{\text {opt }}$ is fairly stable for a wide range of bandwidth allocations: $R_{\text {opt }}(0.5) / R_{\text {opt }}\left(\alpha_{\text {opt }}\right) \approx 0.87$, with $\alpha_{\text {opt }} \approx 0.91$.

On the other hand, when $\gamma=2$ (meaning that users' valuation of delay is eight times higher than in the previous case) we have that $R_{\text {opt }}(0.5) / R_{\text {opt }}\left(\alpha_{\text {opt }}\right) \approx$ 0.66 , i.e., the "loss" incurred by operating the network at a safe bandwidth allocation is higher than in the $\gamma=0.25$ case. In other words, the model predicts that revenues are higher and fairly insensitive to bandwidth allocation when users are more tolerant of delay (which is an intuitively appealing result).

Notice also how the optimal allocation $\alpha_{\text {opt }}$ is dependent on $\gamma$, going from $\alpha_{\text {opt }} \approx 0.91$ to $\alpha_{\text {opt }} \approx 0.67$. In practical terms, this means that an ISP would have to operate its network on the "safe" side (i.e., with $\alpha$ close to 0.5 ) if the value of $\gamma$ cannot be accurately estimated.

\section{Comparison with the case of a single network}

An interesting question is how well a PMP network performs with respect to a one-tiered, non-PMP network. We will begin by presenting a numerical example, followed by a mathematical demonstration of the results that were observed.

\subsection{Numerical comparison}

We are interested in comparing the performance of the two-tiered $(I=2)$ case to that of the one-tiered $(I=1)$ case, under the same set of conditions, both in terms of revenue and network performance measures like delay and input rate. We will revisit here the numerical example studied in Section 4, under the assumption of an exponentially-distributed utility.

We will suppose that both the one-tiered network and the two-tiered PMP network have the same link capacity $c$; in the latter case, this capacity is shared among subnetworks according to Eq. (6). This would correspond to the scenario in which an ISP wants to evaluate the consequences of introducing PMP in its network by deploying the appropriate mechanisms in routers (e.g., packet classification and scheduling mechanisms), but without increasing the installed capacity. 

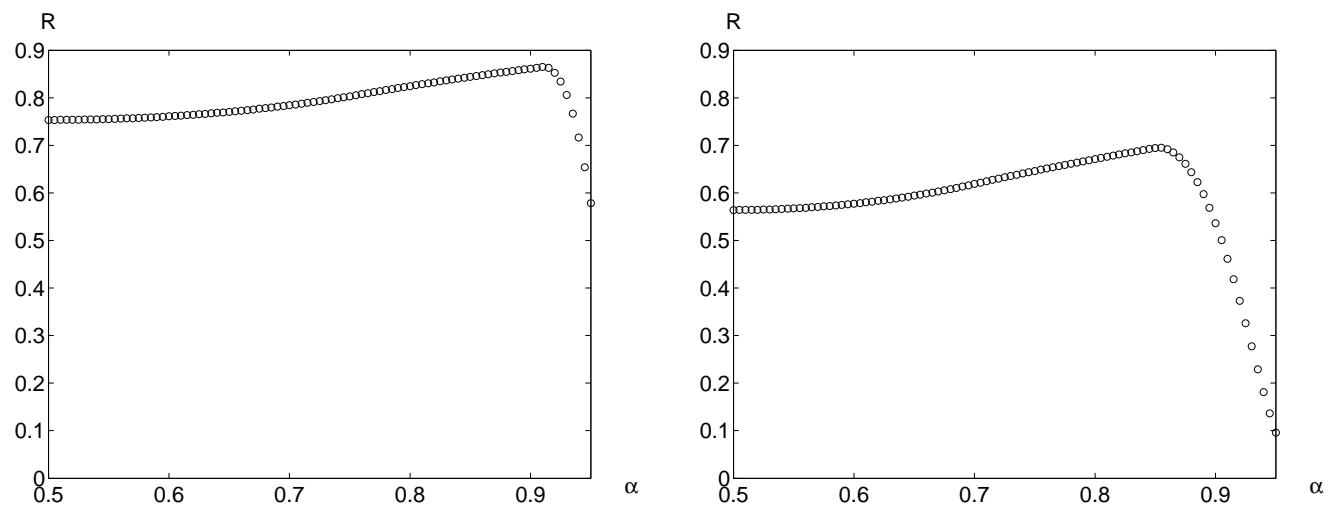

(a) $\gamma=0.25$

(b) $\gamma=0.5$
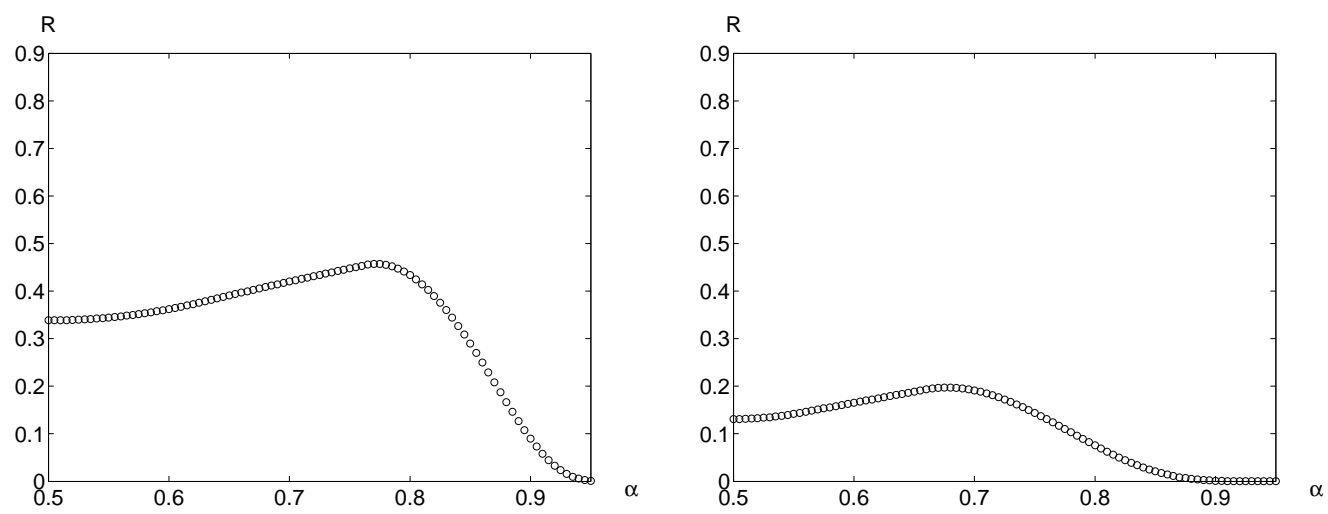

(c) $\gamma=1$

(d) $\gamma=2$

Figure 14: Effect of the $\gamma$ parameter on the optimal revenue, with an exponentially-distributed utility.

$\mathrm{RR} \mathrm{n}^{\circ} 4775$ 
Let us recall the parameters used in this example:

- Total capacity of the network: $c=2$.

- Potential total arrival rate: $\tilde{\lambda}=3$.

- Utility: exponentially distributed, with mean $\bar{U}=1$.

- Cost per unit of delay: $\gamma=1$.

- Service time distribution: exponential.

For the $I=2$ case, Figs. 2, 4, 5, 8 and 10 illustrate the equilibrium region, delay in subnetworks 1 and 2, total cost and revenue, respectively, as a function of the prices $p_{1}$ and $p_{2}$.

We will only present here the results for a bandwidth partition $\alpha=0.7$, because similar results were obtained when using other values of $\alpha$.

Figure 15 plots the revenue $R$, the total cost $p_{t o t}$, per-queue delays and input rates, for both the $I=1$ case and the $I=2$ case. In the latter, for the sake of clarity we show the values of these as a function of $p_{1}$, for a single value of $p_{2}$ such that the curve of $R$ contains the maximum revenue $R_{\text {opt }}$. Hence, the curve for $I=2$ corresponds to a "slice" of the surface depicted in Fig. 10, taken at $p_{2} \approx 0.4$.

Remark 5. Note that, in Fig. 15, the range of values for which the revenue and other performance measures are shown is larger in the $I=1$ case than in the $I=2$ case. This is due to the fact that an equilibrium condition (in the sense of Eqs. (2) and (4)) must be satisfied in the PMP network, which restricts the allowed values for the $p_{i}$, while the single network does not face such equilibrium problems.

Observe that the maximum revenue $R_{\text {opt }}$ is lower (Fig. 15(a)) and the total cost $p_{\text {tot }}$ (Fig. 15(b)) is higher when $I=2$. Total delays, shown in Fig. 15(c), are higher in both PMP subnetworks. Both per-queue $\left(\lambda_{i}\right)$ and total $(\lambda)$ input rates are lower when $I=2$ (Fig. 15(d)), which accounts for the lower revenues.

Note also that, even if we choose the partition parameter $\alpha$ maximizing the revenue for $I=2$ in Fig. 14(c), the revenue is still lower than the optimal one obtained for $I=1$ (Fig. 15(a)). 


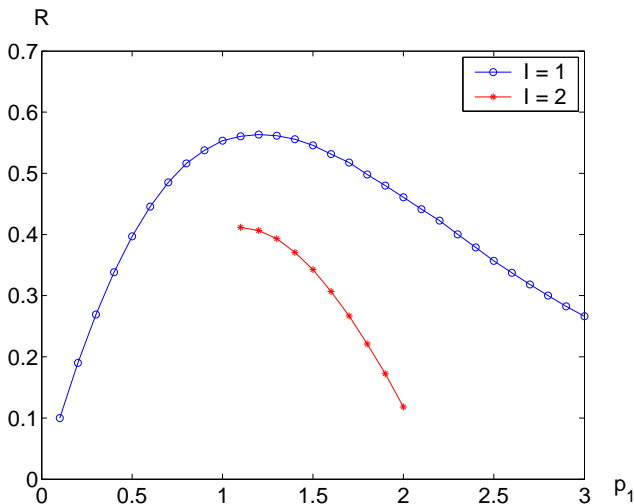

(a) Revenue $R$

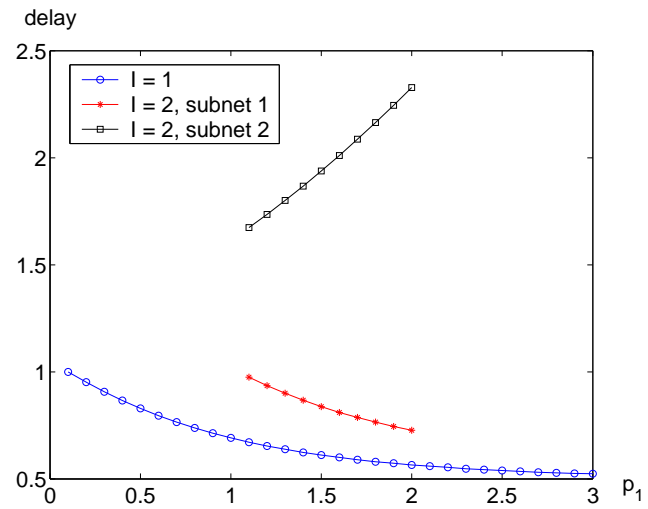

(c) Delay $d$

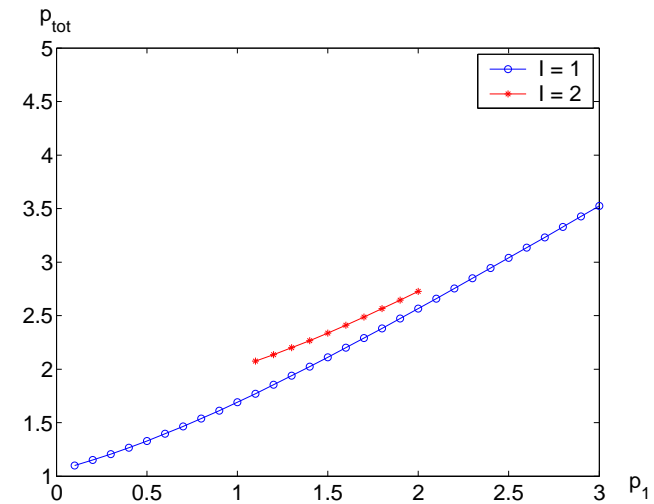

(b) Total cost $p_{t o t}$

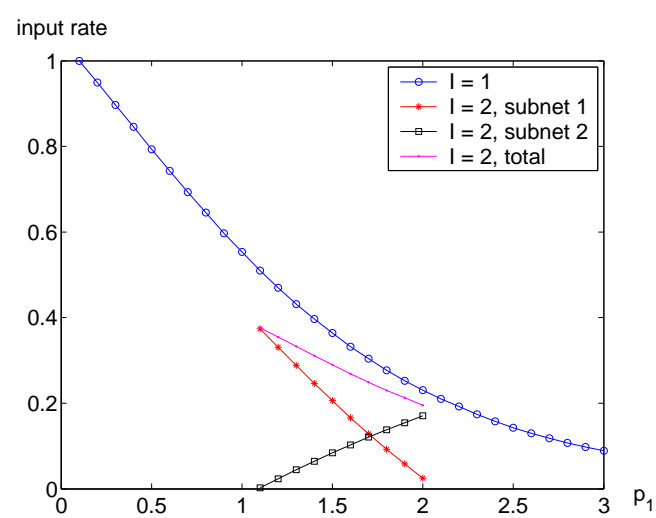

(d) Input rate $\lambda$

Figure 15: Performance as a function of the price $p_{1}$, for $I=1$ (single network) and $I=2$ (PMP).

$\mathrm{RR} \mathrm{n}^{\circ} 4775$ 


\subsection{Formal comparison}

We observed in our preliminary numerical experiments that (somewhat surprisingly) the revenue generated by a single network $(I=1)$ is greater than the revenue generated by two subnetworks $(I=2)$ when the total network capacity - i.e., the service rate - is unchanged, regardless of the partition parameter $\alpha$. Let us formally prove this result for the $\mathrm{M} / \mathrm{M} / 1$ queue in the following theorem.

Theorem 2. Consider a $M / M / 1$ queue (representing the network) with service rate $\mu$ and the response time as the delay cost. The revenue generated by this single queue is greater than the revenue generated by two separate queues with service rates $\alpha \mu$ and $(1-\alpha) \mu$, respectively.

Proof: Consider first the case $I=1$ with a price $p$ per packet. Since the response time $d=1 /(\mu-\lambda)$ is also given by $\left(p_{t o t}-p\right) / \gamma$, we can write $\lambda=\mu-\frac{\gamma}{p_{\text {tot }}-p}$, which yields the stability equation

$$
\bar{F}^{-1}\left(p_{t o t}\right)=\frac{1}{\tilde{\lambda}}\left(\mu-\frac{\gamma}{p_{t o t}-p}\right) .
$$

In the case $I=2$ with prices $p_{1}$ and $p_{2}$, we have

$$
\begin{aligned}
\bar{F}^{-1}\left(p_{t o t}\right) & =\frac{1}{\tilde{\lambda}}\left(\alpha \mu-\frac{\gamma}{p_{t o t}-p_{1}}+(1-\alpha) \mu-\frac{\gamma}{p_{t o t}-p_{2}}\right) \\
& =\frac{1}{\tilde{\lambda}}\left(\mu-\frac{\gamma}{p_{t o t}-p_{1}}-\frac{\gamma}{p_{t o t}-p_{2}}\right) .
\end{aligned}
$$

The proof of the theorem is in two steps: we first show that, for a given $p_{\text {tot}}$, the revenue generated is greater in the case $I=1$; next, we show that, for all $p_{\text {tot }}$ obtained in the case $I=2$, there exists a $p$ in the case $I=1$ giving the same $p_{t o t}$.

Hence, for a given $p_{\text {tot }}$, from the above equations in $p_{\text {tot }}$ we have that, necessarily,

$$
\frac{1}{p_{t o t}-p}=\frac{1}{p_{t o t}-p_{1}}+\frac{1}{p_{t o t}-p_{2}}
$$


that is,

$$
p_{2}=p_{t o t}-\frac{\left(p_{t o t}-p_{1}\right)\left(p_{t o t}-p\right)}{p-p_{1}} .
$$

As $p_{2} \geq 0$ and $p, p_{1}, p_{2} \leq p_{\text {tot }}$, we necessarily have that $p \geq p_{1}$. By symmetry, we also get that $p \geq p_{2}$. Since, for a given $p_{t o t}$, the total arrival rate is the same in both the $I=1$ and the $I=2$ cases, we obtain that the revenue $\left(\lambda_{1}+\lambda_{2}\right) p$ in the case $I=1$ is greater than $\lambda_{1} p_{1}+\lambda_{2} p_{2}$ in the case $I=2$.

Consider now a $p_{\text {tot }}$ obtained in the case $I=2$. Then a price

$$
p=p_{\text {tot }}-\frac{1}{\frac{1}{p_{\text {tot }}-p_{1}}+\frac{1}{p_{\text {tot }}-p_{2}}}
$$

gives the same value of $p_{\text {tot }}$ in the case $I=1$. The only thing that remains to be proven is that such a $p$ is non negative. Since $1 /\left(p_{\text {tot }}-p_{1}\right)+1 /\left(p_{\text {tot }}-p_{2}\right)>1 / p_{\text {tot }}$, this is always the case.

Remark 6. This result can be related to the well-known problem of "splitting" a server in two. Such separation introduces a supplementary mean waiting time as one of the two servers may by idle while the other has some customers waiting for service, something that does not occur in the single-server case (see, for instance, [13]).

Remark 7. The same result applies if we consider the waiting time instead of the response time. Moreover, in the former, the revenue generated in the case $I=2$ tends to the revenue in the case $I=1$ if $\alpha$ tends to 1 . The reason why this does not happen when considering the response time is that the first class (which gets almost all the service rate when a gets close to 1) has to match the total cost of the second class, which tends to infinity with its mean service time $1 /((1-\alpha) \mu)$.

\section{Extensions}

\subsection{A multi-application extension}

Let us now consider different kinds of applications/customers, each having different requirements, i.e., utility variables. Let $K$ be the number of such

$\mathrm{RR} \quad \mathrm{n}^{\circ} 4775$ 
classes. Note that we will use the superscript $k$ for denoting application classes, which are different from the $P M P$ classes - i.e., subnetworks - noted by the subscript $i$.

Instead of a total potential arrival rate $\tilde{\lambda}$ and a utility random variable $U$, we now have a collection of such inputs $\left(\tilde{\lambda}^{(k)}, U^{(k)}\right)$ for $1 \leq k \leq K$.

Denote by $\lambda^{(k)}$ the total arrival rate of packets for application $k, \lambda_{i}^{(k)}$ the arrival of such packets in subnetwork $i$ and, as before, $\lambda_{i}$ the total arrival rate in subnetwork $i$. We have $\lambda_{i}=\sum_{k=1}^{K} \lambda_{i}^{(k)}$ and $\lambda^{(k)}=\sum_{i=1}^{I} \lambda_{i}^{(k)}$.

The set of Wardrop equilibrium equations (1) (i.e., stability conditions) is now

$$
\left\{\begin{aligned}
\lambda^{(k)}=\sum_{i=1}^{I} \lambda_{i}^{(k)} & =\tilde{\lambda}^{(k)} P\left(U^{(k)} \geq p_{\text {tot }}\right) & & \text { for } 1 \leq k \leq K \\
p_{i}+\gamma d_{i} & =p_{\text {tot }} & & \text { for } 1 \leq i \leq I
\end{aligned}\right.
$$

with $d_{i}=f_{i}\left(\lambda_{i}\right)$.

Remark 8. Unicity, in a strict sense, will not be valid anymore since two different application classes can exchange their (or some of their) traffic without modifying Eqs. (8).

Formally, consider a solution of (8) such that $\lambda_{i_{1}}^{\left(k_{1}\right)}, \lambda_{i_{2}}^{\left(k_{1}\right)}, \lambda_{i_{1}}^{\left(k_{2}\right)}, \lambda_{i_{2}}^{\left(k_{2}\right)}>0$, with $k_{1}, k_{2} \in\{1, \cdots, K\}$ and $i_{1}, i_{2} \in\{1, \cdots, I\}$. Then replacing $\lambda_{i_{1}}^{\left(k_{1}\right)}$ by $\lambda_{i_{1}}^{\prime\left(k_{1}\right)}$, $\lambda_{i_{2}}^{\left(k_{1}\right)}$ by $\lambda_{i_{2}}^{\prime\left(k_{1}\right)}=\lambda_{i_{1}}^{\left(k_{1}\right)}+\lambda_{i_{2}}^{\left(k_{1}\right)}-\lambda_{i_{1}}^{\prime\left(k_{1}\right)}, \lambda_{i_{1}}^{\left(k_{2}\right)}$ by $\lambda_{i_{1}}^{\prime\left(k_{2}\right)}=\lambda_{i_{1}}^{\left(k_{1}\right)}+\lambda_{i_{1}}^{\left(k_{2}\right)}-\lambda_{i_{1}}^{\prime\left(k_{1}\right)}$ and $\lambda_{i_{2}}^{\left(k_{2}\right)}$ by $\lambda_{i_{2}}^{\prime\left(k_{2}\right)}=\lambda_{i_{1}}^{\left(k_{2}\right)}+\lambda_{i_{2}}^{\left(k_{2}\right)}-\lambda_{i_{1}}^{\prime\left(k_{2}\right)}$, still provides a solution of (8) because this system depends only on $\lambda^{(k)}$ and $\lambda_{i} \forall k, i$ and those total rates are kept unchanged.

Nonetheless, we have the following result on the existence and unicity of equilibrium, in terms of $p_{t o t}, \lambda^{(k)}$ and $\lambda_{i} \forall k, i$ :

Proposition 1. Assume that $p_{1}>\cdots>p_{I}$. Existence and unicity of equilibrium is verified, i.e., the $\lambda^{(k)}$ and $\lambda_{i} \forall k, i$ exist and are unique provided that

$$
\sum_{k=1}^{K} \tilde{\lambda}^{(k)} \bar{F}^{(k)}\left(p_{1}\right) \geq \sum_{i=1}^{I} f_{i}^{-1}\left(\frac{p_{1}-p_{i}}{\gamma}\right)
$$


Proof: We have $\forall 1 \leq k \leq K$,

$$
p_{\text {tot }}=\left(\bar{F}^{(k)}\right)^{-1}\left(\lambda^{(k)} / \tilde{\lambda}^{(k)}\right)
$$

and $\forall 1 \leq i \leq I$,

$$
p_{t o t}=p_{i}+\gamma d_{i} .
$$

Note that, according to (10), $\lambda^{(k)}$ decreases continuously when $p_{\text {tot }}$ increases, so that $\lambda=\sum_{k=1}^{K} \lambda^{(k)}$ decreases continuously when $p_{\text {tot }}$ increases.

Also, according to (11), $\lambda_{i}$ increases continuously when $p_{\text {tot }}$ increases, so that $\lambda=\sum_{i=1}^{I} \lambda_{i}$ increases continuously with $p_{\text {tot }}$. Thus, there exists a unique $p_{\text {tot }}$ such that both $\lambda$ (obtained from Eq. (10) and (11)) are equal, as long as the value of $\sum_{k=1}^{K} \lambda^{(k)}$ (from Eq. (10)) is greater that the value of $\sum_{i=1}^{I} \lambda_{i}$ (from Eq. (11)) when $p_{\text {tot }}=p_{1}=\max \left\{p_{i}\right\}$. This gives condition (9).

$\lambda_{i}$ and $\lambda^{(k)} \forall k, i$ immediately follow.

Note that the strict non-unicity result is not a problem for computing the revenue since the objective function depends only on $\lambda_{i}$ and $p_{i}$, and not directly on $\lambda_{i}^{(k)}$.

Remark 9. The multi-application case can be seen as a single-application case if, instead of considering the set $\left\{\left(\tilde{\lambda}^{(k)}, F^{(k)}\right) \mid 1 \leq k \leq K\right\}$ with $F^{(k)}$ the cumulative distribution of random variable $U^{(k)}$, we consider the "mixture"

$$
\left(\sum_{k=1}^{K} \tilde{\lambda}^{(k)}, \frac{\sum_{k=1}^{K} \tilde{\lambda}^{(k)} F^{(k)}}{\sum_{k=1}^{K} \tilde{\lambda}^{(k)}}\right) .
$$

\subsection{A multi-application extension with per-application delay valuations}

\subsubsection{Model description and analysis}

Now let us suppose that, as in the previous subsection, there are $K$ types of applications/customers, but with a delay valuation $\gamma^{(k)}$ for the class- $k$ application. In this case, the set of stability equations (1) becomes

$\mathrm{RR} \mathrm{n}^{\circ} 4775$ 


$$
\left\{\begin{aligned}
\lambda^{(k)}=\sum_{i=1}^{I} \lambda_{i}^{(k)} & =\tilde{\lambda}^{(k)} P\left(U^{(k)} \geq p_{\text {tot }}^{(k)}\right) & & \text { for } 1 \leq k \leq K, \\
p_{i}+\gamma^{(k)} d_{i} & =p_{\text {tot }}^{(k)} & & \text { for } 1 \leq i \leq I \text { and } 1 \leq k \leq K
\end{aligned}\right.
$$

with $d_{i}=f_{i}\left(\lambda_{i}\right)$.

This means that the set (12) has to be verified for all $k$, but with a different $p_{t o t}$ for each $k$. Note however that the equations cannot be solved independently $\forall k$ since they are related through the queueing delays $d_{i}(1 \leq i \leq I)$.

Remark 10. In this case, the system behaves like a game where each player $k$ (i.e., each application) tries to stabilize its traffic, that is, it tries to equalize $p_{i}+\gamma^{(k)} d_{i}$ for all $i$, meaning that $p_{\text {tot }}^{(k)}$ exists. In this sense, a solution of (12), if it exists, is a Nash equilibrium between all the classes.

Proving the existence and unicity of the solution is more complicate in this case, since we do not have a single $p_{t o t}$, so that the proof in Section 6.1 does not stand anymore. Therefore, finding out conditions for existence and unicity is still an open question.

Nevertheless, based on the results in previous sections we can still find a necessary, but not sufficient, condition for equilibrium.

Proposition 2. Assume that $p_{1} \geq p_{2} \geq \cdots \geq p_{I}$. A necessary condition for solving (12) is that

$$
\sum_{k=1}^{K} \tilde{\lambda}^{(k)} \bar{F}^{(k)}\left(p_{1}\right) \geq \sum_{i=1}^{I} f_{i}^{-1}\left(\frac{p_{1}-p_{i}}{\gamma^{(k)}}\right) .
$$

Proof: We have, $\forall k$,

$$
p_{\text {tot }}^{(k)}=\left(\bar{F}^{(k)}\right)^{-1}\left(\frac{\lambda^{(k)}}{\tilde{\lambda}^{(k)}}\right) .
$$

If (1) is verified, then we also have that, $\forall i$,

$$
p_{\text {tot }}^{(k)}=p_{i}+\gamma^{(k)} d_{i}
$$


From (14), we can see that $\lambda^{(k)}$ decreases when $p_{\text {tot }}^{(k)}$ increases, so $\lambda=\sum_{k=1}^{K} \lambda^{(k)}$ decreases when $p_{\text {tot }}^{(k)}$ increases. From (15), we see that $\lambda_{i}$ increases when $p_{\text {tot }}^{(k)}$ increases, so $\lambda=\sum_{i=1}^{I} \lambda_{i}$ increases as well. In order to ensure that both quantities be equal, the inequality given in the proposition must hold when $p_{\text {tot }}^{(k)}=\max _{i} p_{i}=p_{1}$, meaning that $\sum_{k=1}^{K} \lambda^{(k)}=\sum_{i=1}^{I} \lambda_{i}$ at the minimum $p_{\text {tot }}^{(k)}$ value.

The reason why this proof does not provide a sufficient condition for the existence and unicity of equilibrium is that, in the proof, we look at the existence of $p_{t o t}^{(k)}$ for a given $k$, and not at the existence of the $p_{\text {tot }}^{(k)}$ all together.

\subsubsection{Numerical example}

We will present a numerical example that illustrates the problems described in Section 6.2.1. Let us consider a two-tiered PMP network $(I=2)$ with $K=2$ application classes having different delay valuations $\gamma^{(k)}$; we will suppose that application 1 is more delay-sensitive than application 2 . The parameters take the following values:

- Total capacity of the network: $c=10$.

- Bandwidth partition: $\alpha=2 / 3$.

- Potential total arrival rate, per application: $\tilde{\lambda}^{(1)}=\tilde{\lambda}^{(2)}=10$.

- Utility: exponentially distributed, with mean $\bar{U}^{(1)}=1$ and $\bar{U}^{(2)}=2$.

- Cost per unit of delay: $\gamma^{(1)}=2, \gamma^{(2)}=1$.

- Service time distribution: exponential.

The results below were obtained by trying to solve Eq. (12) for $\lambda^{(k)}, \lambda_{i}$ and $p_{\text {tot }}^{(k)}$, with $k=1,2$ and $i=1,2$, while taking into account the necessary condition (13). A solution of (12) was searched for every price pair $\left(p_{1}, p_{2}\right)$ such that $p_{i}=0.05 \cdot n$ with $n=1, \ldots, 40$ and $i=1,2$.

Figure 16(a) shows, for every pair of prices, whether the necessary condition (13) is satisfied or not, whereas Fig. 16(b) indicates whether the set (12) could be solved or not - that is, whether $p_{t o t}^{(k)}, k=1,2$ could be found. Each

$\mathrm{RR} \mathrm{n}^{\circ} 4775$ 
square on the grid corresponds to a given $\left(p_{1}, p_{2}\right)$. A gray square denotes that the condition is satisfied and that the $p_{\text {tot }}^{(k)}$ are found, respectively, while white squares denote the opposite situation.

The gray region in Fig. 16(a) is equivalent to the equilibrium region in the single-application case. Note however that, for some prices $\left(p_{1}, p_{2}\right)$ satisfying (13), a solution to (12) could not be found: these are the white "holes" in the gray region of Fig. 16(b).

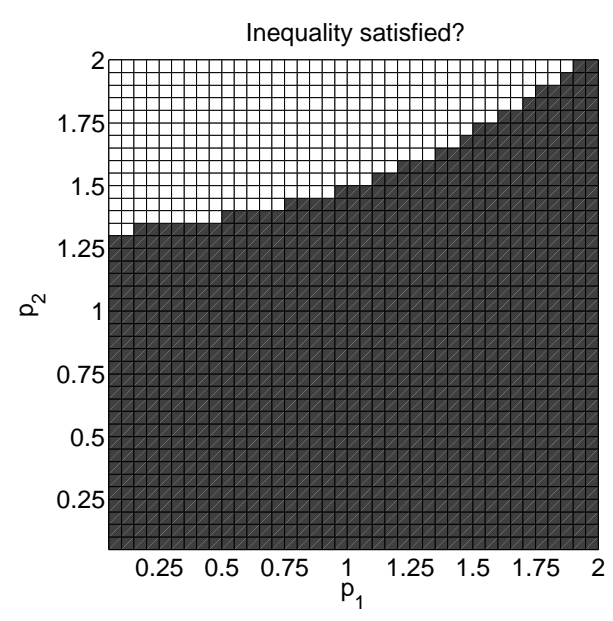

(a) Necessary condition for equilibrium.

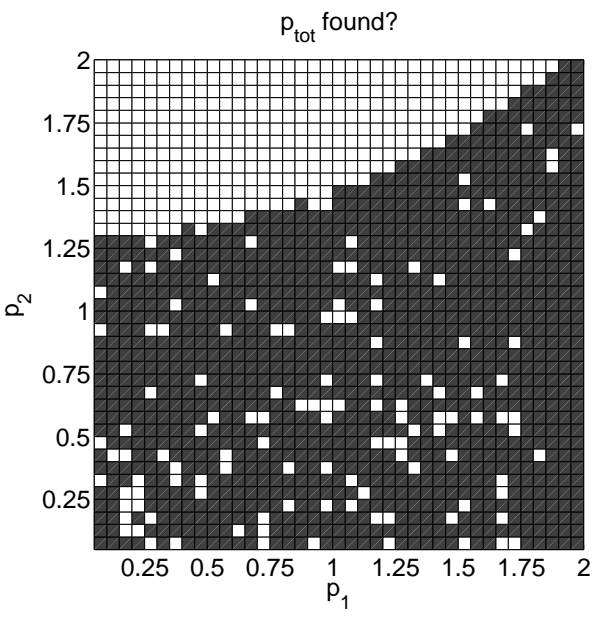

(b) The set of equations (12) may be solved.

Figure 16: Per-application delay valuations: an example.

The sparsity of the set of solutions illustrates the difficulty of finding a necessary and sufficient condition over the set of prices, corresponding to the general difficulty of finding out conditions for Nash equilibrium. 


\subsection{A model including losses and delays as QoS require- ments}

Another interesting extension of our model would be to consider other QoS metrics than delay only. We consider here that quality, as perceived by a user, is a mixture of delay and loss probability.

For class $i$, the actual total cost function is

$$
p_{i}+\gamma d_{i}+\zeta B_{i}
$$

where $B_{i}$ is the loss probability (for class $i$ ) and $\zeta$ is a constant converting loss in money. A packet enters network $i$ if $i=\operatorname{argmin}_{j} p_{j}+\gamma d_{j}+\zeta B_{j}$ and $U \geq p_{i}+\gamma d_{i}+\zeta B_{i}$.

In equilibrium, $p_{\text {tot }}=p_{i}+\gamma d_{i}+\zeta B_{i}, \forall i$. The actual total arrival rate is still

$$
\lambda=\tilde{\lambda} P\left(U \geq p_{\text {tot }}\right) .
$$

The system of equations (1) becomes

$$
\left\{\begin{aligned}
\sum_{i=1}^{I} \lambda_{i} & =\tilde{\lambda} P\left(U \geq p_{\text {tot }}\right) \\
p_{i}+\gamma d_{i}+\zeta B_{i} & =p_{\text {tot }}, \text { for } 1 \leq i \leq I
\end{aligned}\right.
$$

where $d_{i}=f_{i}\left(\lambda_{i}\right)$ and $B_{i}=g_{i}\left(\lambda_{i}\right)$ depend both on $\lambda_{i}$. Define the function $h_{i}$ as $h_{i}=\gamma f_{i}+\zeta g_{i}$.

For simplicity, we consider here only the waiting time; extending this model to take into account the response time is straightforward.

$p_{\text {tot }}$ is then given by the equation:

$$
p_{t o t}=\bar{F}^{-1}\left(\frac{\sum_{i=1}^{I} h_{i}^{-1}\left(p_{t o t}-p_{i}\right)}{\tilde{\lambda}}\right)
$$

and, $\forall 1 \leq i \leq I$,

$$
\lambda_{i}=h_{i}^{-1}\left(p_{t o t}-p_{i}\right)
$$

$\mathrm{RR} \mathrm{n}^{\circ} 4775$ 
Proposition 3. Assume that $p_{1}>\cdots>p_{I}$. The system of equations (16) has a unique solution if and only if

$$
\left.p_{1} \leq \bar{F}^{-1}\left(\frac{1}{\tilde{\lambda}} \sum_{i=1}^{I} h_{i}^{-1}\left(p_{1}-p_{i}\right)\right)\right) .
$$

This corresponds to the stability condition described in Section 3.

Proof: the proof follows closely that of Theorem 1. Functions $f_{i}$ and $g_{i}$ are strictly increasing functions of $\lambda_{i}$. Then, $h_{i}$ and $h_{i}^{-1}$ are also strictly increasing. Following the arguments of Theorem 1, the left-hand side of Eq. 17 is strictly increasing whereas the right-hand side is decreasing which means that, if a solution exists, it is unique. By the same kind of arguments than in Theorem 1, a necessary and sufficient condition for existence is Eq. (18).

Thus, including losses in the model does not add any theoretical complexity. Even if inverting $h_{i}$ analytically is harder than inverting $f_{i}$ (when considering the case of delay only), it is still numerically simple due to the monotonicity of $h_{i}$.

\subsection{Time-of-day Pricing}

In practice, demand is varying over time. Time-of-day pricing, as practiced for instance in power pricing, is an interesting way to manage the traffic flow.

Time-of-day pricing is modelled as follows. We assume that a day is decomposed in different periods of time during which the demand (meaning the utility) follows a relatively constant distribution. In each period $j$ of time, the random variable representing user's utility is $U_{j}$, with cumulative distribution function $F_{j}$. For every $j$, the prices are independently determined like done for problem (1).

\section{Conclusions}

In this paper, we have introduced a mathematical model of the Paris Metro Pricing (PMP) scheme for charging packet networks. This pricing method 
looks convenient for Internet Service Providers since it is fairly easy to implement and deploy in an ISP network using current, off-the-shelf technologies. Even if PMP does not provide strict QoS guarantees, users would probably appreciate it since the total charge is linear in the volume of data, hence predictable.

Our model has allowed us to find some necessary and sufficient conditions on the prices required to obtain an equilibrium. Extensions to the model, allowing to take into account the multi-application case and multiple QoS requirements, as well as time-of-day pricing, are also included. Many numerical illustrations are provided.

The model has pointed out a possible drawback of the PMP scheme, namely, that for a given network capacity revenues may be lower in a network implementing PMP. This limitation, coming from the adopted bandwidthsharing policy among subnetworks, could be alleviated by means of more efficient scheduling mechanisms.

As directions for future research, we are interested in trying to carry out the same kind of analysis for the round-robin scheme of [4] (to tackle the problem of revenue maximization). Also, the case of strict QoS requirements could be investigated.

\section{References}

[1] E. Altman and L. Wynter. Equilibrium, games, and pricing in transportation and telecommunication networks. Technical Report 4632, INRIA, 2002 .

[2] L.A. DaSilva. Pricing of QoS-Enabled Networks: A Survey. IEEE Communications Surveys \& Tutorials, 3(2), 2000.

[3] P. Dolan. Internet Pricing. is the end of the World Wide Wait in view? Communications \& Strategies, 37:15-46, 2000.

[4] P. Dube, V.S. Borkar, and D. Manjunath. Differential join prices for parallel queues: Social optimality, dynamic pricing algorithms and application to internet pricing. In Proceedings of IEEE INFOCOM 02, 2002.

$\mathrm{RR} \mathrm{n}^{\circ} 4775$ 
[5] M. Falkner, M. Devetsikiotis, and I. Lambadaris. An Overview of Pricing Concepts for Broadband IP Networks. IEEE Communications Surveys 8 Tutorials, 3(2), 2000.

[6] P.C. Fishburn and A.M. Odlyzko. Dynamic Behavior of Differential Pricing and Quality of Service Options for the Internet. In Proceedings of ICE98, pages 128-139. ACM, 1998.

[7] R. Gibbens, R. Mason, and R. Steinberg. Internet service classes under competition. IEEE Journal on Selected Areas in Communications, 18(12):2490-2498, 2000.

[8] T. Henderson, J. Crowcroft, and S. Bhatti. Congestion Pricing. Paying Your Way in Communication Networks. IEEE Internet Computing, September/October:85-89, 2001.

[9] M.L. Honig and K. Steiglitz. Usage-based pricing of packet data generated by a heterogeneous user population. In Proceedings of IEEE INFOCOM 95, pages 867-874, 1995.

[10] A. Odlyzko. Paris Metro Pricing for the Internet. In ACM Conference on Electronic Commerce (EC'99), pages 140-147, 1999.

[11] A. Odlyzko. The history of communications and its implications for the Internet. Technical report, AT\&T Labs, 2000.

[12] B. Stiller, P. Reichl, and S. Leinen. Pricing and Cost Recovery for Internet Services: Practical Review, Classification, and Application of Relevant Models. Netnomics, 2(1), 2000.

[13] K.S. Trivedi. Probability and Statistics with Reliability, Queuing, and Computer Science Applications. John Wiley \& Sons, 2002. Second Edition.

[14] B. Tuffin. Charging the Internet without bandwidth reservation: an overview and bibliography of mathematical approaches. Technical Report 1434, IRISA, January 2002. 


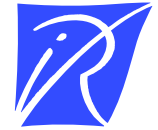

Unité de recherche INRIA Lorraine, Technopôle de Nancy-Brabois, Campus scientifique, 615 rue du Jardin Botanique, BP 101, 54600 VILLERS LÈS NANCY

Unité de recherche INRIA Rennes, Irisa, Campus universitaire de Beaulieu, 35042 RENNES Cedex

Unité de recherche INRIA Rhône-Alpes, 655, avenue de l'Europe, 38330 MONTBONNOT ST MARTIN

Unité de recherche INRIA Rocquencourt, Domaine de Voluceau, Rocquencourt, BP 105, 78153 LE CHESNAY Cedex

Unité de recherche INRIA Sophia-Antipolis, 2004 route des Lucioles, BP 93, 06902 SOPHIA-ANTIPOLIS Cedex

Éditeur

INRIA, Domaine de Voluceau, Rocquencourt, BP 105, 78153 LE CHESNAY Cedex (France)

http://www.inria.fr

ISSN 0249-6399 